\title{
SCHUR COMPLEMENT PRECONDITIONERS FOR SURFACE INTEGRAL-EQUATION FORMULATIONS OF DIELECTRIC PROBLEMS SOLVED WITH THE MULTILEVEL FAST MULTIPOLE ALGORITHM*
}

\author{
TAHIR MALAS ${ }^{\dagger}$ AND LEVENT GÜREL ${ }^{\dagger}$
}

\begin{abstract}
Surface integral-equation methods accelerated with the multilevel fast multipole algorithm (MLFMA) provide a suitable mechanism for electromagnetic analysis of real-life dielectric problems. Unlike the perfect-electric-conductor case, discretizations of surface formulations of dielectric problems yield $2 \times 2$ partitioned linear systems. Among various surface formulations, the combined tangential formulation $(\mathrm{CTF})$ is the closest to the category of first-kind integral equations, and hence it yields the most accurate results, particularly when the dielectric constant is high and/or the dielectric problem involves sharp edges and corners. However, matrix equations of CTF are highly ill-conditioned, and their iterative solutions require powerful preconditioners for convergence. Second-kind surface integral-equation formulations yield better conditioned systems, but their conditionings significantly degrade when real-life problems include high dielectric constants. In this paper, for the first time in the context of surface integral-equation methods of dielectric objects, we propose Schur complement preconditioners to increase their robustness and efficiency. First, we approximate the dense system matrix by a sparse near-field matrix, which is formed naturally by MLFMA. The Schur complement preconditioning requires approximate solutions of systems involving the $(1,1)$ partition and the Schur complement. We approximate the inverse of the $(1,1)$ partition with a sparse approximate inverse (SAI) based on the Frobenius norm minimization. For the Schur complement, we first approximate it via incomplete sparse matrix-matrix multiplications, and then we generate its approximate inverse with the same SAI technique. Numerical experiments on sphere, lens, and photonic crystal problems demonstrate the effectiveness of the proposed preconditioners. In particular, the results for the photonic crystal problem, which has both surface singularity and a high dielectric constant, shows that accurate CTF solutions for such problems can be obtained even faster than with second-kind integral equation formulations, with the acceleration provided by the proposed Schur complement preconditioners.
\end{abstract}

Key words. preconditioning, sparse-approximate-inverse preconditioners, partitioned matrices, Schur complement reduction method, integral-equation methods, dielectric problems, computational electromagnetics

AMS subject classifications. 31A10, 65B99, 65F10, 65R20, 65Y20, 78A45, 78A55, 78M05

DOI. $10.1137 / 090780808$

1. Introduction. We consider preconditioning of dense, complex, and nonHermitian linear systems, which are obtained by discretizing surface integral-equation formulations of dielectric problems. These linear systems have an explicit $2 \times 2$ partitioned structure in the form

$$
\left[\begin{array}{ll}
\overline{\boldsymbol{A}}_{11} & \overline{\boldsymbol{A}}_{12} \\
\overline{\boldsymbol{A}}_{21} & \overline{\boldsymbol{A}}_{22}
\end{array}\right] \cdot\left[\begin{array}{l}
\boldsymbol{x}_{J} \\
\boldsymbol{x}_{M}
\end{array}\right]=\left[\begin{array}{l}
\boldsymbol{b}_{1} \\
\boldsymbol{b}_{2}
\end{array}\right], \text { or } \overline{\boldsymbol{A}} \cdot \boldsymbol{x}=\boldsymbol{b},
$$

*Submitted to the journal's Methods and Algorithms for Scientific Computing section December 21, 2009; accepted for publication (in revised form) January 5, 2011; published electronically October 4, 2011. This work was supported by the Scientific and Technical Research Council of Turkey (TUBITAK) under research grant 107E136, the Turkish Academy of Sciences in the framework of the Young Scientist Award Program (LG/TUBA-GEBIP/2002-1-12), and by contracts from ASELSAN and SSM.

http://www.siam.org/journals/sisc/33-5/78080.html

${ }^{\dagger}$ Department of Electrical and Electronics Engineering, Bilkent University, TR-06800, Bilkent, Ankara, Turkey, and Computational Electromagnetics Research Center (BiLCEM), Bilkent University, TR-06800, Bilkent, Ankara, Turkey (tmalas@ee.bilkent.edu.tr, lgurel@bilkent.edu.tr).

2440 
where

$$
\overline{\boldsymbol{A}} \in \mathbb{C}^{2 N \times 2 N} \quad \text { and } \quad \overline{\boldsymbol{A}}_{11}, \overline{\boldsymbol{A}}_{12}, \overline{\boldsymbol{A}}_{21}, \overline{\boldsymbol{A}}_{22} \in \mathbb{C}^{N \times N} .
$$

In (1.1), $\boldsymbol{x}_{J}$ and $\boldsymbol{x}_{M}$ are $N \times 1$ coefficient vectors of the Rao-Wilton-Glisson (RWG) [49] basis functions expanding the equivalent electric and magnetic electric currents, respectively, and $\boldsymbol{b}_{1,2}$ represent $N \times 1$ excitation vectors obtained by testing incident fields.

We analyze four types of surface formulations that are commonly used in computational electromagnetics (CEM): the combined tangential formulation $(\mathrm{CTF})$, the combined normal formulation (CNF), the modified normal Müller formulation (MNMF), and the electric and magnetic current combined-field integral equation (JMCFIE) (which is derived from the combination of CTF and CNF) [59,60,61]. Many real-life problems in CEM involve dielectrics, such as the development of effective lenses [47], simulations of photonic crystals [36], and optical analysis of blood for blood-related diseases [41].

For large-scale problems, preconditioning is a vital technique for increasing the robustness and efficiency of iterative solvers [4]. As is commonly known, a preconditioner is a matrix $\overline{\boldsymbol{M}}$ that approximates the system matrix $\overline{\boldsymbol{A}}$, and for which it is not expensive to find the solution vector $\boldsymbol{v}$ of

$$
\bar{M} \cdot \boldsymbol{v}=\boldsymbol{w}
$$

for a given right-hand-side vector $\boldsymbol{w}$. In this paper, we aim a right-preconditioned system by solving

$$
\overline{\boldsymbol{A}} \cdot \overline{\boldsymbol{M}}^{-1} \cdot \boldsymbol{y}=\boldsymbol{b} \quad \text { with } \quad \boldsymbol{x}=\overline{\boldsymbol{M}}^{-1} \cdot \boldsymbol{y}
$$

instead of the original system (1.1). As the preconditioner $\overline{\boldsymbol{M}}$ approximates the system matrix $\overline{\boldsymbol{A}}$ better, we expect fewer iterations for convergence. On the other hand, the costs of both construction and application of the preconditioner increase with better approximations. Hence, a balance should be maintained between the approximation level and preconditioning costs so that the preconditioned system is solved in less time compared to the unpreconditioned one.

Note that standard algebraic preconditioners that do not take into account the partitioned structure often perform poorly on systems similar to (1.1). Discretizations of surface formulations yield indefinite matrices that are far from diagonally dominant, especially for high dielectric constants [62]. Therefore, incomplete-LU-type (ILUtype) preconditioners may exhibit instability problems, or very slow convergence [4]. Surface integral formulations of CEM give rise to off-diagonal partitions that are much weaker than diagonal ones; hence it is also difficult to find suitable nonzero patterns for sparse approximate inverses (SAIs).

In the literature, preconditioning techniques for systems similar to (1.1) are usually studied in the context of generalized saddle-point problems $[2,5,6,13,16,23$, $35,46,52,54,63]$. By approximating the dense system matrix in (1.1) by a sparse near-field matrix, preconditioners developed for saddle-point problems can be adapted to integral-equation formulations of dielectric problems. The partitions in (1.1), however, do not satisfy any of the conditions that generally exist in saddle-point problems, such as symmetry or positive definiteness [6]. Moreover, contrary to our case, in many applications that lead to partitioned systems, the $(2,2)$ partition is zero or has a much smaller dimension than other partitions. In general, preconditioners are 
tailored depending on the specific properties of the underlying problem [6]. Hence, preconditioners developed for other applications may not be readily applicable to surface integral-equation formulations.

In this work, we consider preconditioners that are obtained with some approximations to Schur complement reduction. We use the sparse near-field matrix to construct preconditioners. The near-field matrix is formed naturally in the context of the multilevel fast multipole algorithm (MLFMA), which is employed to accelerate the dense matrix-vector multiplications (MVMs). The success of the Schur complement preconditioners depends on effective approximations for the solutions of systems involving the $(1,1)$ partition and the Schur complement. Similar to the work in [13], the current paper uses SAIs in these approximations. In [13], however, the authors use an iterative method [15] to generate the sparsity pattern of an SAI in the course of construction. In our case, the near-field pattern is a natural candidate for the sparsity pattern of an SAI, and this approach leads to successful preconditioners for the surface integralequation formulations of perfect-electric-conductor (PEC) objects [10, 43]. Therefore, we employ the Frobenius-norm minimization technique and use the available nearfield pattern for approximate inverses. The advantages of using SAIs over ILU-type preconditioners are robustness and ease of parallelization. Furthermore, by using the block structure of the near-field matrix, we eliminate the high setup time of SAI. The approximation for the Schur complement is more delicate than the $(1,1)$ partition. In the literature, most of the proposed approaches are limited to cases in which the $(2,2)$ partition is zero. We propose to obtain an approximate Schur complement via incomplete matrix-matrix multiplications that retain the near-field sparsity pattern. Then we construct an SAI from the approximate Schur complement.

This paper is organized as follows: In section 2, we briefly summarize integral equation formulations of dielectric problems and the structure of MLFMA. Then we introduce the Schur complement reduction method and related preconditioners. We discuss approximations for the $(1,1)$ partition and the Schur complement in section 4. In the numerical results section, we compare proposed preconditioners with simple and ILU-type preconditioners using sphere and two real-life problems: a lens and a photonic crystal.

A note on the use of "partitions" and "blocks." Throughout the paper, we will use the term partition to denote one of the submatrices of a $2 \times 2$ partitioned system, i.e., we call $\overline{\boldsymbol{A}}_{11}$ in $(1.1)$ the $(1,1)$ partition of $\overline{\boldsymbol{A}}$. As will be detailed in section 3 , partitions of the near-field matrix are composed of interactions between pairs of neighboring lowest-level MLFMA clusters. In the CEM community, the term block is used to denote these interactions. We will adopt this convention and imply building blocks of a near-field partition by the term block.

2. Surface integral-equation methods for dielectric problems. The surface integral-equation approach is an important class of numerical methods in electromagnetics scattering analyses of three-dimensional (3-D) dielectric objects having arbitrary shapes [48]. Recently, significant progress has been made in devising new formulations that are well suited for iterative solutions [59, 60, 61]. In this section, we will briefly review these methods.

For all formulations, consider a closed homogeneous dielectric object that resides in a homogeneous medium. Let the electric permittivity and the electric permeability of the outer region of the object be $\epsilon_{1}, \mu_{1}$, and let those of the inner region be $\epsilon_{2}, \mu_{2}$, respectively. Using the equivalence principle, an equivalent electric current $\boldsymbol{J}$ and an 
equivalent magnetic current ${ }^{1} M$ are defined on the surface $S$ of the object. Depending on the testing procedure and the considered electromagnetic field, various integralequation formulations can be derived.

2.1. The combined tangential formulation (CTF). If the boundary condition on the surface is tested directly, tangential electric-field and magnetic-field integral equations for the outer and the inner regions can be defined. For example, the tangential electric-field integral equation (T-EFIE) for the outer region is defined as [34]

$$
\hat{\boldsymbol{t}} \cdot \eta_{1} \mathcal{T}_{1}\{\boldsymbol{J}\}-\hat{\boldsymbol{t}} \cdot \mathcal{K}_{1}\{\boldsymbol{M}\}-\hat{\boldsymbol{t}} \cdot \frac{1}{2} \hat{\boldsymbol{n}} \times \boldsymbol{M}=-\hat{\boldsymbol{t}} \cdot \boldsymbol{E}^{i n c} \quad(\mathrm{~T}-\mathrm{EFIE-O}),
$$

where $\hat{\boldsymbol{t}}$ is any tangential vector on the surface, $\eta_{1}=\sqrt{\mu_{1} / \epsilon_{1}}$ is the impedance of the outer medium,

$$
\mathcal{T}_{l}\{\boldsymbol{X}\}=i k_{l} \int_{S} d \boldsymbol{r}^{\prime}\left[\boldsymbol{X}\left(\boldsymbol{r}^{\prime}\right)+\frac{1}{k_{l}^{2}} \nabla^{\prime} \cdot \boldsymbol{X}\left(\boldsymbol{r}^{\prime}\right) \nabla\right] g_{l}\left(\boldsymbol{r}, \boldsymbol{r}^{\prime}\right)
$$

and

$$
\mathcal{K}_{l}\{\boldsymbol{X}\}=\int_{P V, S} d \boldsymbol{r}^{\prime} \boldsymbol{X}\left(\boldsymbol{r}^{\prime}\right) \times \nabla^{\prime} g_{l}\left(\boldsymbol{r}, \boldsymbol{r}^{\prime}\right)
$$

are the operators that can be defined for both the outer $(l=1)$ and inner $(l=2)$ regions, $\hat{\boldsymbol{n}}$ is the outward normal vector on the surface $S$, and $\boldsymbol{E}^{i n c}$ is the incident electric field on the object. In (2.2) and (2.3), $k_{l}$ is the wavenumber in the corresponding medium, $P V$ is the principal value of the integral, and

$$
g_{l}\left(\boldsymbol{r}, \boldsymbol{r}^{\prime}\right)=\frac{e^{i k_{l}\left|\boldsymbol{r}-\boldsymbol{r}^{\prime}\right|}}{4 \pi\left|\boldsymbol{r}-\boldsymbol{r}^{\prime}\right|}
$$

is the scalar Green's function of the 3-D scalar Helmholtz equation for medium $l$, which represents the response at $\boldsymbol{r}$ due to a point source located at $\boldsymbol{r}^{\prime}$. For the inner region, the tangential electric-field integral equation is

$$
\hat{\boldsymbol{t}} \cdot \eta_{2} \mathcal{T}_{2}\{\boldsymbol{J}\}-\hat{\boldsymbol{t}} \cdot \mathcal{K}_{2}\{\boldsymbol{M}\}+\hat{\boldsymbol{t}} \cdot \frac{1}{2} \hat{\boldsymbol{n}} \times \boldsymbol{M}=0 \quad \text { (T-EFIE-I), }
$$

where $\eta_{2}$ is the impedance of the inner medium. Similar equations can also be obtained by testing the tangential magnetic fields. Respectively, the tangential magnetic-field integral equation (T-MFIE) for the outer and inner regions are

$$
\hat{\boldsymbol{t}} \cdot \frac{1}{\eta_{1}} \mathcal{T}_{1}\{\boldsymbol{M}\}+\hat{\boldsymbol{t}} \cdot \mathcal{K}_{1}\{\boldsymbol{J}\}+\hat{\boldsymbol{t}} \cdot \frac{1}{2} \hat{\boldsymbol{n}} \times \boldsymbol{J}=-\hat{\boldsymbol{t}} \cdot \boldsymbol{H}^{i n c} \quad(\text { T-MFIE-O) }
$$

and

$$
\hat{\boldsymbol{t}} \cdot \frac{1}{\eta_{2}} \mathcal{T}_{2}\{\boldsymbol{M}\}+\hat{\boldsymbol{t}} \cdot \mathcal{K}_{2}\{\boldsymbol{J}\}-\hat{\boldsymbol{t}} \cdot \frac{1}{2} \hat{\boldsymbol{n}} \times \boldsymbol{J}=0 \quad \text { (T-MFIE-I) }
$$

\footnotetext{
${ }^{1}$ Preconditioning matrices $(\overline{\boldsymbol{M}})$ and magnetic currents $(\boldsymbol{M})$ are denoted by similar symbols, following conventions. Since one of them is a matrix $(\overline{\boldsymbol{M}})$ and the other one is a vector $(\boldsymbol{M})$, they should be clearly distinguishable from the context.
} 
The four sets of integral equations, i.e., (2.1), (2.5), (2.6), and (2.7), can be combined in several ways to solve for the unknown currents $\boldsymbol{J}$ and $\boldsymbol{M}$ [48]. In particular, the combination of the outer and the inner equations produces internal-resonance-free formulations. Among such formulations, we consider the recently proposed CTF [61], which is defined as

$$
\begin{aligned}
& \frac{1}{\eta_{1}} \text { T-EFIE-O }+\frac{1}{\eta_{2}} \text { T-EFIE-I, } \\
& \eta_{1} \text { T-MFIE-O }+\eta_{2} \text { T-MFIE-I. }
\end{aligned}
$$

Note that the identity terms in (2.8) (implicit in the MFIE operators) are not well tested, and the resulting matrices are, in general, ill-conditioned and far from being diagonally dominant. Hence, CTF is closer to the category of first-kind integral equation. Also note that $\boldsymbol{J}$ is well tested in T-EFIE and $\boldsymbol{M}$ is well tested in TMFIE [61], hence the combination used in CTF leads to a stable matrix equation. The scaling of the tangential equations further improves the condition of the formulation compared to its former variants [61], such as the tangential Poggio-Miller-ChangHarrington-Wu-Tsai formulation [11, 57].

2.2. The combined normal formulation (CNF). Although CTF produces a stable formulation, it still suffers from slow convergence since it is closer to a firstkind integral equation. Hence, several authors proposed second-kind and betterconditioned integral-equation formulations by making use of the normal formulations [62]. These formulations can be obtained by testing the fields after they are projected onto the surface via a cross-product by $\hat{\boldsymbol{n}}$. The normal outer and inner electric-field integral equations are, respectively,

$$
-\hat{\boldsymbol{n}} \times \eta_{1} \mathcal{T}_{1}\{\boldsymbol{J}\}+\hat{\boldsymbol{n}} \times \mathcal{K}_{1}\{\boldsymbol{M}\}-\frac{1}{2} \boldsymbol{M}=\hat{\boldsymbol{n}} \times \boldsymbol{E}^{i n c} \quad(\mathrm{~N}-\mathrm{EFIE}-\mathrm{O})
$$

and

$$
\hat{\boldsymbol{n}} \times \eta_{2} \mathcal{T}_{2}\{\boldsymbol{J}\}-\hat{\boldsymbol{n}} \times \mathcal{K}_{2}\{\boldsymbol{M}\}-\frac{1}{2} \boldsymbol{M}=0 \quad \text { (N-EFIE-I) }
$$

For the magnetic field, normal formulations yield

$$
\hat{\boldsymbol{n}} \times \frac{1}{\eta_{1}} \mathcal{T}_{1}\{\boldsymbol{M}\}+\hat{\boldsymbol{n}} \times \mathcal{K}_{1}\{\boldsymbol{J}\}-\frac{1}{2} \boldsymbol{J}=-\hat{\boldsymbol{n}} \times \boldsymbol{H}^{i n c} \quad(\mathrm{~N}-\mathrm{MFIE}-\mathrm{O})
$$

and

$$
-\hat{\boldsymbol{n}} \times \frac{1}{\eta_{2}} \mathcal{T}_{2}\{\boldsymbol{M}\}-\hat{\boldsymbol{n}} \times \mathcal{K}_{2}\{\boldsymbol{J}\}-\frac{1}{2} \boldsymbol{J}=0 \quad(\mathrm{~N}-\mathrm{MFIE}-\mathrm{I}) .
$$

Then, similar to CTF, CNF is formed by the linear combinations of the outer and inner integral equations, i.e.,

$$
\begin{array}{r}
\text { N-MFIE-O + N-MFIE-I, } \\
\text { N-EFIE-O + N-EFIE-I. }
\end{array}
$$

However, the identity terms do not cancel out in CNF, and a second-kind integral equation is obtained. When the Galerkin scheme is used to discretize (2.13), these well-tested identity operators appear on the diagonal partitions of the coefficient matrix, resulting in more diagonally dominant linear systems than tangential formulations. 
2.3. The modified normal Müller formulation (MNMF). In [60], the authors show that a scaled version of the normal Müller formulation [45] leads to a well-conditioned and stable formulation. Later, it is shown by the same authors that MNMF produces the lowest iteration counts for iterative solutions of dielectric problems compared to other stable formulations. Hence, we also consider MNMF, which is actually a scaled version of CNF. MNMF is defined as [60]

$$
\begin{gathered}
\frac{\mu_{1}}{\mu_{1}+\mu_{1}} \text { N-MFIE-O }+\frac{\mu_{2}}{\mu_{1}+\mu_{1}} \text { N-MFIE-I, } \\
\frac{\epsilon_{1}}{\epsilon_{1}+\epsilon_{1}} \text { N-EFIE-O }+\frac{\epsilon_{2}}{\epsilon_{1}+\epsilon_{1}} \text { N-EFIE-I. }
\end{gathered}
$$

2.4. The electric and magnetic current combined-field integral formulation (JMCFIE). For nondielectric PEC metallic objects, a combination of the electric-field integral equation and the magnetic-field integral equation yields the combined-field integral equation [50], which has favorable characteristics for iterative solutions [53]. In the dielectric case, a similar combination of CTF and CNF can be formed as [59]

$$
\mathrm{JMCFIE}=\alpha \mathrm{CTF}+\beta \mathrm{CNF},
$$

where $0 \leq \alpha \leq 1$ and $\beta=1-\alpha$. Similar to the PEC case, the matrix systems of the JMCFIE formulation are more stable and can usually be solved in fewer iterations compared to those of $\mathrm{CTF}$ and $\mathrm{CNF}$ [22].

2.5. Comparison of the integral-equation formulations for dielectrics. All of the aforementioned integral-equation formulations have pros and cons in terms of storage, accuracy, and conditioning. In terms of memory use, CTF requires the least memory when MLFMA is applied to the solution. The reason is that CTF has identical diagonal partitions and the same set of far-field patterns for the inner and outer regions. CNF and JMCFIE also have identical diagonal partitions, but they have different far-field patterns for each region. Finally, in addition to having different far-field patterns, MNMF also has different diagonal partitions due to different scaling of N-MFIE-O and N-EFIE-I in (2.14). These differences between the formulations can be remarkable, because the storage of the near-field matrix and the radiation patterns constitute the highest memory requirements in MLFMA. For example, the solution of a sphere geometry with approximately 413,000 unknowns leads to 1.1 GB difference of memory use between CTF and MNMF [22]. In that example, the sphere has a radius of $7.5 \lambda$, where $\lambda$ denotes the wavelength in free space.

$\mathrm{CTF}$ is closer to a first-kind integral-equation formulation, whereas the other formulations (CNF, MNMF, and JMCFIE) are all second-kind formulations. In CTF, the singularity of the hypersingular operator $\mathcal{T}$ can be decreased by moving the differential operator from the Green's function to the testing function. Hence, CTF has a smoothing kernel, in contrast to other formulations with singular kernels [62]. The smoothing property of the CTF kernel results in coefficient matrices that are far from being diagonally dominant and that have poor conditioning. On the other hand, due to the smoothing property of its kernel, CTF has a better solution accuracy compared to normal formulations (CNF and MNMF). JMCFIE includes CNF, and therefore is also less accurate than CTF. Despite the accuracy drawbacks, the singular kernels and the identity terms of normal formulations and JMCFIE lead to more diagonally dominant matrices and better conditioning than CTF.

Copyright $@$ ㅇ by SIAM. Unauthorized reproduction of this article is prohibited. 
To evaluate the integral-equation formulations, however, one should also consider two important parameters that seriously affect the accuracy and the stability of the resulting matrices: the dielectric constant (or relative permittivity) of the medium $\left(\epsilon_{r}=\epsilon_{2} / \epsilon_{1}\right)$ and the shape of the geometry. Both the solution accuracy and the conditioning of second-kind integral equations decrease as the dielectric constant increases [62]. Irregularities of the geometry, i.e., surfaces having sharp edges and corners, also have a negative effect on the accuracy of second-kind integral equations. Therefore, when the dielectric constant is high and/or the surface of the object has nonsmooth sections, the accuracy of second-kind integral equations can be much poorer than the accuracy of CTF [62]. Finally, integral equations of the second kind are also shown to be more sensitive to discretization quality of the surface and to the accuracy of the numerical integration than integral equations of the first kind.

From these discussions, it can be deduced that preconditioning is a critical issue for accurate and efficient electromagnetics simulations of dielectric objects. When the surface of the object has nonsmooth regions or the dielectric constant of the object is high, the accuracy of second-kind equations can be unacceptable and one may have to employ CTF, for which the solutions are tough to obtain without effective preconditioning. Moreover, a high dielectric constant impairs the conditioning of normal formulations, and this can necessitate applying effective preconditioners to these formulations.

3. Discretization of surface integral-equation formulations and MLFMA. We can denote the surface integral equations described in section 2 as

$$
\begin{array}{r}
\mathcal{L}_{11}\{\boldsymbol{J}\}+\mathcal{L}_{12}\{\boldsymbol{M}\}=\boldsymbol{G}_{1}, \\
\mathcal{L}_{21}\{\boldsymbol{J}\}+\mathcal{L}_{22}\{\boldsymbol{M}\}=\boldsymbol{G}_{2}
\end{array}
$$

using linear operators $\mathcal{L}_{k l}$. Projecting each operator in (3.1) onto the $N$-dimensional space $\operatorname{span}\left\{\boldsymbol{f}_{1}, \boldsymbol{f}_{2}, \ldots, \boldsymbol{f}_{N}\right\}$ formed by the divergence-conforming RWG testing functions [49], we have

$$
\begin{aligned}
& \left\langle\boldsymbol{f}_{m}, \mathcal{L}_{11}\{\boldsymbol{J}\}\right\rangle+\left\langle\boldsymbol{f}_{m}, \mathcal{L}_{12}\{\boldsymbol{M}\}\right\rangle=\left\langle\boldsymbol{f}_{m}, \boldsymbol{G}_{1}\right\rangle, \quad 1 \leq m \leq N, \\
& \left\langle\boldsymbol{f}_{m}, \mathcal{L}_{21}\{\boldsymbol{J}\}\right\rangle+\left\langle\boldsymbol{f}_{m}, \mathcal{L}_{22}\{\boldsymbol{M}\}\right\rangle=\left\langle\boldsymbol{f}_{m}, \boldsymbol{G}_{2}\right\rangle,
\end{aligned}
$$

where

$$
\langle\boldsymbol{f}, \boldsymbol{g}\rangle=\int d \boldsymbol{r} f(\boldsymbol{r}) \cdot \boldsymbol{g}(\boldsymbol{r})
$$

denotes the inner product of two real-valued vector functions $\boldsymbol{f}$ and $\boldsymbol{g}$. This process is also known as "testing the integral equation." By choosing the basis functions to be the same as the testing functions, we adopt a Galerkin scheme and seek the discrete solutions of

$$
\boldsymbol{J} \approx \sum_{n=1}^{N} x_{J_{n}} \boldsymbol{f}_{n}
$$

and

$$
\boldsymbol{M} \approx \sum_{n=1}^{N} x_{M n} \boldsymbol{f}_{n}
$$

Copyright $@$ by SIAM. Unauthorized reproduction of this article is prohibited. 
in the same $N$-dimensional space. As a result, the complex-valued coefficient vectors $\boldsymbol{x}_{J}$ and $\boldsymbol{x}_{M}$ become the solution of the $2 N \times 2 N$ linear system

$$
\left[\begin{array}{ll}
\overline{\boldsymbol{A}}_{11} & \overline{\boldsymbol{A}}_{12} \\
\overline{\boldsymbol{A}}_{21} & \overline{\boldsymbol{A}}_{22}
\end{array}\right] \cdot\left[\begin{array}{l}
\boldsymbol{x}_{J} \\
\boldsymbol{x}_{M}
\end{array}\right]=\left[\begin{array}{l}
\boldsymbol{b}_{1} \\
\boldsymbol{b}_{2}
\end{array}\right]
$$

where

$$
\left(\overline{\boldsymbol{A}}_{k l}\right)_{m n}=\left\langle\boldsymbol{f}_{m}, \mathcal{L}_{k l}\left\{\boldsymbol{f}_{n}\right\}\right\rangle, \quad\left(\boldsymbol{b}_{i}\right)_{m}=\left\langle\boldsymbol{f}_{m}, \boldsymbol{G}_{i}\right\rangle, \quad k, l=1,2, \quad m, n=1,2, \ldots, N .
$$

Since the RWG basis functions are defined on planar triangles, geometry surfaces are discretized accordingly, i.e., via planar triangulation. Each basis function is associated with an edge; hence the number of unknowns is equal to the total number of edges in a mesh. Unless dictated by the geometry, we set the average size of an edge about one-tenth of the wavelength as a rule of thumb.

Many real-life problems require the analysis of objects that have sizes on the order of several wavelengths. Therefore, the solution of the dense system (1.1) can only be obtained by iterative solvers, which make use of the fast methods, such as MLFMA. In MLFMA, MVMs of each partition in (3.6) are performed in $\mathcal{O}\left(N N_{L}\right)$ computational complexity, where $N_{L}=\mathcal{O}(\log N)$ [12]. For this purpose, a tree structure of $N_{L}$ levels is constructed by positioning the dielectric object in a cube and then recursively dividing the cube into smaller ones, which are called clusters. On any level, clusters that do not touch each other are assigned as far-field clusters and the others as nearfield clusters. The interactions among touching lowest-level clusters constitute the near-field matrix, whose entries are calculated directly using numerical integration techniques $[18,25,30,58]$ and stored in the memory for later use in MVMs. In this way, the dense system matrix is decomposed into its far-field and near-field parts as

$$
\left[\begin{array}{ll}
\overline{\boldsymbol{A}}_{11} & \overline{\boldsymbol{A}}_{12} \\
\overline{\boldsymbol{A}}_{21} & \overline{\boldsymbol{A}}_{22}
\end{array}\right]=\left[\begin{array}{ll}
\overline{\boldsymbol{A}}_{11}^{N F} & \overline{\boldsymbol{A}}_{12}^{N F} \\
\overline{\boldsymbol{A}}_{21}^{N F} & \overline{\boldsymbol{A}}_{22}^{N F}
\end{array}\right]+\left[\begin{array}{ll}
\overline{\boldsymbol{A}}_{11}^{F F} & \overline{\boldsymbol{A}}_{12}^{F F} \\
\overline{\boldsymbol{A}}_{21}^{F F} & \overline{\boldsymbol{A}}_{22}^{F F}
\end{array}\right] \text {, or } \overline{\boldsymbol{A}}=\overline{\boldsymbol{A}}^{N F}+\overline{\boldsymbol{A}}^{F F} .
$$

Since the lowest-level cluster is fixed to a certain size (i.e., $0.25 \lambda$ ) and the number of touching clusters is also fixed by the shape of the geometry, there are $\mathcal{O}(N)$ near-field interactions in each partition. In addition, the clustering of the geometry leads to a near-field matrix with block-structured partitions, where the blocks of partitions correspond to interactions of the lowest-level near-field clusters [42].

Interactions of the far-field clusters are computed by employing MLFMA individually for each partition of the system matrix. MLFMA performs a matrix-vector multiplication, where the matrix elements are the interactions between pairs of far-field clusters, in a group-by-group and multilevel manner via processes called aggregation, translation, and disaggregation. In the aggregation stage, radiation patterns of the basis functions are multiplied with the excitation coefficients (i.e., the input vector of the iterative solver), and radiated fields of the higher-level clusters are calculated in a bottom-up scheme in the tree structure. Between two consecutive levels, interpolations are employed to match the different sampling rates of the fields using a local interpolation method [20,21]. For each pair of far-field clusters, their cluster-to-cluster interaction is computed in the translation stage. In any specific level, translations are performed only for clusters whose parents are in the near-field zone of each other. Interactions with farther clusters are accounted for by the translations of higher levels. Because of the cubic symmetry, the number of translation operators is $\mathcal{O}(1)$ for 
each level. In the disaggregation stage, a top-down computation scheme is followed to find the total incoming fields at the cluster centers. Translations and incoming fields of parent clusters are combined to find the total incoming field for each cluster. Transpose interpolations (or anterpolations) [9] are employed to reduce the sampling rates of the fields of parent clusters in order to adapt them as incoming fields of child clusters. The matrix-vector multiplication is completed in the lowest level when the incoming fields are shifted from the centers of the clusters onto the testing functions, and inner products are computed in the form of spectral integrations.

4. Preconditioning with approximate Schur complement reduction. For iterative solutions of partitioned linear systems, preconditioners are frequently based on segregated methods. In such methods, the unknown vectors are computed separately [6]. The main representative of the segregated approach is the Schur complement reduction method. Since the whole matrix is not explicitly available in our case, we first approximate the dense system matrix with the sparse near-field matrix, i.e.,

$$
\overline{\boldsymbol{A}} \approx \overline{\boldsymbol{A}}^{N F} .
$$

In general, magnitudes of the elements of the matrix $\overline{\boldsymbol{A}}$ change with physical proximity [43]. Therefore, the near-field matrix $\overline{\boldsymbol{A}}^{N F}$ is likely to preserve the most relevant contributions of the dense system matrix.

4.1. Schur complement reduction. Consider the $2 \times 2$ partitioned near-field system,

$$
\left[\begin{array}{ll}
\overline{\boldsymbol{A}}_{11}^{N F} & \overline{\boldsymbol{A}}_{12}^{N F} \\
\overline{\boldsymbol{A}}_{21}^{N F} & \overline{\boldsymbol{A}}_{22}^{N F}
\end{array}\right] \cdot\left[\begin{array}{l}
\boldsymbol{v}_{1} \\
\boldsymbol{v}_{2}
\end{array}\right]=\left[\begin{array}{l}
\boldsymbol{w}_{1} \\
\boldsymbol{w}_{2}
\end{array}\right],
$$

which can be rewritten as

$$
\begin{aligned}
& \overline{\boldsymbol{A}}_{11}^{N F} \cdot \boldsymbol{v}_{1}+\overline{\boldsymbol{A}}_{12}^{N F} \cdot \boldsymbol{v}_{2}=\boldsymbol{w}_{1}, \\
& \overline{\boldsymbol{A}}_{21}^{N F} \cdot \boldsymbol{v}_{1}+\overline{\boldsymbol{A}}_{22}^{N F} \cdot \boldsymbol{v}_{2}=\boldsymbol{w}_{2} .
\end{aligned}
$$

When $\overline{\boldsymbol{A}}_{11}^{N F}$ is nonsingular, from (4.3)

$$
\boldsymbol{v}_{1}=\left(\overline{\boldsymbol{A}}_{11}^{N F}\right)^{-1} \cdot\left(\boldsymbol{w}_{1}-\overline{\boldsymbol{A}}_{12}^{N F} \cdot \boldsymbol{v}_{2}\right) .
$$

If we insert (4.5) in (4.4) and rearrange, we can find $\boldsymbol{v}_{2}$ from

$$
\overline{\boldsymbol{S}} \cdot \boldsymbol{v}_{2}=\boldsymbol{w}_{2}-\overline{\boldsymbol{A}}_{21}^{N F} \cdot\left(\overline{\boldsymbol{A}}_{11}^{N F}\right)^{-1} \cdot \boldsymbol{w}_{1}
$$

where

$$
\overline{\boldsymbol{S}}=\overline{\boldsymbol{A}}_{22}^{N F}-\overline{\boldsymbol{A}}_{21}^{N F} \cdot\left(\overline{\boldsymbol{A}}_{11}^{N F}\right)^{-1} \cdot \overline{\boldsymbol{A}}_{12}^{N F}
$$

is the Schur complement. Once $\boldsymbol{v}_{2}$ is found from (4.6), $\boldsymbol{v}_{1}$ can be found using

$$
\overline{\boldsymbol{A}}_{11}^{N F} \cdot \boldsymbol{v}_{1}=\boldsymbol{w}_{1}-\overline{\boldsymbol{A}}_{12}^{N F} \cdot \boldsymbol{v}_{2} .
$$

Schur complement reduction is an attractive solution technique if the order of the Schur complement $\overline{\boldsymbol{S}}$ is small and if linear systems with matrix $\overline{\boldsymbol{A}}_{11}^{N F}$ can be solved efficiently. Even when these requirements are not entirely satisfied, approximate solutions of (4.6) and (4.8) can serve as useful preconditioners. Hence, we consider the approximate solution of the system (4.2) as an important step of constructing and applying a preconditioner. 
4.2. Preconditioners based on approximate Schur complement reduction. Next, we describe four types of preconditioners derived from the Schur complement reduction with different approximations to the solutions of (4.8) and (4.6) [6].

4.2.1. Diagonal approximate Schur preconditioner (DASP). The diagonal approximate Schur preconditioner (DASP) is derived with the approximations

$$
\overline{\boldsymbol{A}}_{12}^{N F}=\overline{\boldsymbol{A}}_{12}^{N F} \approx 0
$$

performed on the right-hand sides (RHSs) of (4.8) and (4.6). Then these equations reduce to

$$
\overline{\boldsymbol{A}}_{11}^{N F} \cdot \boldsymbol{v}_{1}=\boldsymbol{w}_{1}
$$

and

$$
\overline{\boldsymbol{S}} \cdot \boldsymbol{v}_{2}=\boldsymbol{w}_{2}
$$

Therefore, the preconditioning matrix of DASP is given by

$$
\overline{\boldsymbol{M}}_{D A S P}=\left[\begin{array}{cc}
\overline{\boldsymbol{A}}_{11}^{N F} & 0 \\
0 & \overline{\boldsymbol{S}}
\end{array}\right] .
$$

4.2.2. Upper triangular approximate Schur preconditioner (UTASP). If we set only one of the off-diagonal partitions $\overline{\boldsymbol{A}}_{12}^{N F}$ and $\overline{\boldsymbol{A}}_{21}^{N F}$ in the RHSs of (4.8) and (4.6) to zero, we obtain a partition triangular preconditioner. When we set $\overline{\boldsymbol{A}}_{21}^{N F} \approx 0$, we obtain the upper triangular approximate Schur preconditioner (UTASP). First, we have to solve for $\boldsymbol{v}_{2}$ from

$$
\overline{\boldsymbol{S}} \cdot \boldsymbol{v}_{2}=\boldsymbol{w}_{2}
$$

Then we can find $\boldsymbol{v}_{1}$ using $\boldsymbol{v}_{2}$ :

$$
\overline{\boldsymbol{A}}_{11}^{N F} \cdot \boldsymbol{v}_{1}=\boldsymbol{w}_{1}-\overline{\boldsymbol{A}}_{12}^{N F} \cdot \boldsymbol{v}_{2} .
$$

Given the same RHS, UTASP finds the same $\boldsymbol{v}_{2}$ with DASP, but it is expected to compute a more accurate $\boldsymbol{v}_{1}$. The preconditioning matrix of UTASP is defined as

$$
\overline{\boldsymbol{M}}_{U T A S P}=\left[\begin{array}{cc}
\overline{\boldsymbol{A}}_{11}^{N F} & \overline{\boldsymbol{A}}_{12}^{N F} \\
0 & \overline{\boldsymbol{S}}
\end{array}\right] .
$$

4.2.3. Lower triangular approximate Schur preconditioner (LTASP). If we set $\overline{\boldsymbol{A}}_{12}^{N F} \approx 0$ instead of $\overline{\boldsymbol{A}}_{21}^{N F}$, we obtain the lower triangular approximate Schur preconditioner (LTASP). In this case, we have to first solve for $\boldsymbol{v}_{1}$ from

$$
\overline{\boldsymbol{A}}_{11}^{N F} \cdot \boldsymbol{v}_{1}=\boldsymbol{w}_{1} \text {. }
$$

Then we can find $\boldsymbol{v}_{2}$ using $\boldsymbol{v}_{1}$ :

$$
\overline{\boldsymbol{S}} \cdot \boldsymbol{v}_{2}=\boldsymbol{w}_{2}-\overline{\boldsymbol{A}}_{21}^{N F} \cdot\left(\overline{\boldsymbol{A}}_{11}^{N F}\right)^{-1} \cdot \boldsymbol{w}_{1}=\boldsymbol{w}_{2}-\overline{\boldsymbol{A}}_{21}^{N F} \cdot \boldsymbol{v}_{1} .
$$

Compared to DASP, LTASP finds the same $\boldsymbol{v}_{1}$, but it is expected to find a more accurate $\boldsymbol{v}_{2}$ for a given RHS. The preconditioning matrix of LTASP is defined as

$$
\overline{\boldsymbol{M}}_{L T A S P}=\left[\begin{array}{ll}
\overline{\boldsymbol{A}}_{11}^{N F} & 0 \\
\overline{\boldsymbol{A}}_{21}^{N F} & \overline{\boldsymbol{S}}
\end{array}\right] .
$$


4.2.4. Approximate Schur preconditioner (ASP). In an effort to devise an effective preconditioner, it is also an option not to omit any of the off-diagonal blocks in $\overline{\boldsymbol{A}}^{N F}$. For efficiency, however, solutions of the systems involving $\overline{\boldsymbol{S}}$ and $\overline{\boldsymbol{A}}_{11}^{N F}$ should be performed approximately, as will be detailed in section 4.3. Hence, we call this preconditioner the approximate Schur preconditioner (ASP), for which the preconditioning matrix is given by

$$
\overline{\boldsymbol{M}}_{A S P}=\overline{\boldsymbol{A}}^{N F}=\left[\begin{array}{ll}
\overline{\boldsymbol{A}}_{11}^{N F} & \overline{\boldsymbol{A}}_{12}^{N F} \\
\overline{\boldsymbol{A}}_{21}^{N F} & \overline{\boldsymbol{A}}_{22}^{N F}
\end{array}\right] .
$$

4.3. Approximations of the solutions involving $\bar{A}_{11}^{N F}$ and the Schur complement $\overline{\boldsymbol{S}}$. The performance of the preconditioners explained in sections 4.2.1, $4.2 .2,4.2 .3$, and 4.2 .4 depends on the availability of fast and approximate solutions to

$$
\overline{\boldsymbol{A}}_{11}^{N F} \cdot \boldsymbol{v}_{1}=\boldsymbol{w}_{1}^{\prime}
$$

and

$$
\overline{\boldsymbol{S}} \cdot \boldsymbol{v}_{2}=\boldsymbol{w}_{2}^{\prime}
$$

where $\boldsymbol{w}_{1}^{\prime}$ and $\boldsymbol{w}_{2}^{\prime}$ take different forms depending on the type of preconditioner. Since the approximations performed in these solutions define a preconditioner for the linear system (1.1), accurate solutions are not required. On the other hand, very crude approximations of the exact solutions may deteriorate the quality of the preconditioner, and iteration counts may not be decreased as desired.

In the literature, several approximation strategies for the solutions of (4.20) and (4.21) have been proposed, but many of them are strongly problem dependent [6]. For surface integral-equation formulations, we discuss possible approximations and our approach for $\overline{\boldsymbol{A}}_{11}$ and $\overline{\boldsymbol{S}}$.

4.3.1. Approximating the solutions involving $\bar{A}_{11}^{N F}$. For some specific problems, many efficient techniques are available for a fast and accurate solution of (4.20). For example, if the system matrix were obtained from the discretization of a differential operator, in many cases a few multigrid sweeps would yield efficient and yet sufficiently accurate solutions [19]. In general situations, however, one must resort to algebraic approaches, such as ILU factorizations, SAIs, or approximations by a few iterations of a Krylov subspace method.

In this work, we approximate the solution of the system (4.20) by an SAI of $\overline{\boldsymbol{A}}_{11}^{N F}$. We denote the SAI of $\overline{\boldsymbol{A}}_{11}^{N F}$ as $\overline{\boldsymbol{M}}_{11}$. Hence, our approximation becomes

$$
\left(\bar{A}_{11}^{N F}\right)^{-1} \approx \bar{M}_{11}
$$

SAI preconditioners have been successfully used in CEM for PEC problems [1, 10, 39, 43]. Two important advantages of SAI preconditioners over ILU-type preconditioners are robustness and ease of parallelization [8]. In our case, it is also possible to alleviate the high construction cost of SAI using the block structure of the near-field matrix $[10,43]$, as we describe in the following paragraph.

Approximate inverses of sparse matrices can be obtained in several ways $[7,8$, 15, 26, 38]. Among these methods, we make use of the Frobenius-norm technique [8], which decouples the generation of an $N \times N$ SAI into $N$ independent least-squares 

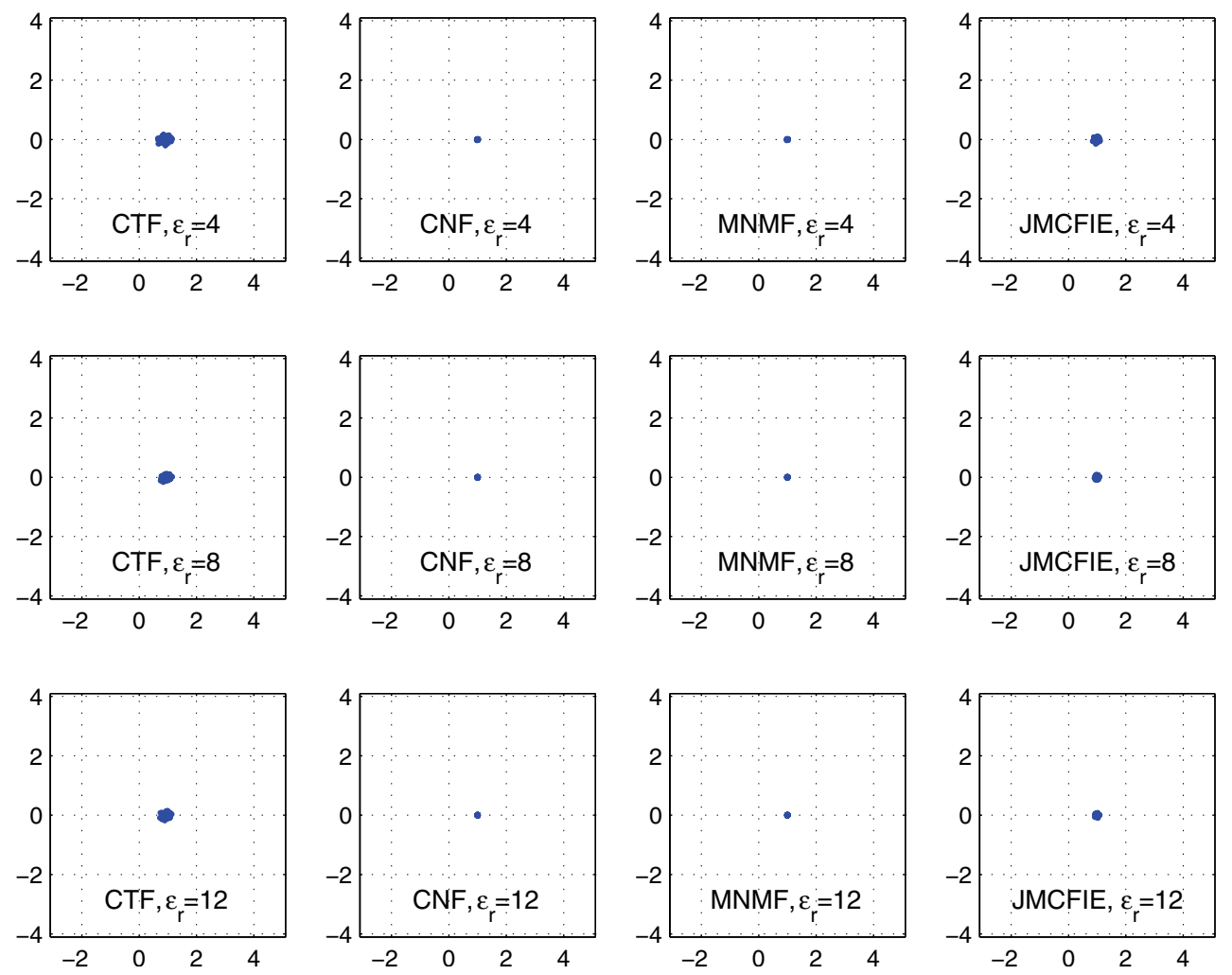

FIG. 1. Eigenvalues of $\overline{\boldsymbol{M}}_{11} \cdot \overline{\boldsymbol{A}}_{11}^{N F}$ for different formulations and increasing dielectric constants of 4,8 , and 12 .

problems for each row. Then each least-squares problem can be solved by employing a QR factorization and an upper triangular system solution [56]. On the other hand, due to the block structure of $\overline{\boldsymbol{A}}_{11}^{N F}$, we need to perform only $N / m$ QR factorizations, where $m$ is the average block size of $\overline{\boldsymbol{A}}_{11}^{N F}$. For a $0.25 \lambda$ lowest-level box size and $\lambda / 10$ mesh size, typical values of $m$ lie between 20 and 50, depending on the geometry. Since the QR factorization constitutes the dominant cost in a least-squares solution, we significantly reduce the construction time of SAI.

We evaluate the approximation (4.22) in Figure 1, where we depict eigenvalues of matrices $\overline{\boldsymbol{M}}_{11} \cdot \overline{\boldsymbol{A}}_{11}^{N F}$ for different formulations and increasing dielectric constants of 4,8 , and 12 . The geometry is a $0.5 \lambda$ sphere involving 1,860 unknowns. We see that eigenvalues are very tightly clustered around $(1,0)$ for normal formulations (CNF and MNMF). For CTF, we see a slightly looser clustering than CNF and MNMF. JMCFIE lies between the two cases. Also note that the spectra of $\overline{\boldsymbol{A}}_{11}^{N F}$ are unaffected by the increase of the dielectric constant.

4.3.2. Approximating the solutions involving $\overline{\boldsymbol{S}}$. The approximation involving the Schur complement matrix $\overline{\boldsymbol{S}}$ is more subtle than that of $\overline{\boldsymbol{A}}_{11}^{N F}$. Moreover, it is shown that the approximation quality provided to the system involving $\overline{\boldsymbol{S}}$ should accommodate the approximation level to the system involving $\overline{\boldsymbol{A}}_{11}^{N F}$ [54]. Therefore, we try to find an approximation for $\overline{\boldsymbol{S}}$ that is as good as the approximation for $\overline{\boldsymbol{A}}_{11}^{N F}$.

In the literature related to saddle-point problems, several choices exist when the 
system matrix $\overline{\boldsymbol{A}}$ is symmetric [6]. These choices include multigrid sweeps and loworder discretization of the related operator. Many purely algebraic approaches have also been proposed for the nonsymmetric case, in which the $(2,2)$ partition is zero. Those approaches include approximating the inverse of the $(1,1)$ partition in the Schur complement by the inverse of the diagonal or block-diagonal part of the $(1,1)$ partition. Better approximations can be provided in the form of incomplete factors (e.g., [40]). However, a limited number of methods exist for the case of a nonzero $(2,2)$ partition $[6,13,54]$. Perhaps one of the most applicable methods is to use a Krylov subspace solver to obtain an approximate solution of the system (4.21). MVMs with $\overline{\boldsymbol{S}}$ can be provided to the solver by multiplications with the $(2,2)$ and offdiagonal partitions, and by another iterative solve with $\overline{\boldsymbol{A}}_{11}^{N F}$. The required solve with $\overline{\boldsymbol{A}}_{11}^{N F}$, however, can significantly increase the application cost of the preconditioner. Moreover, in many cases, a preconditioner for $\overline{\boldsymbol{S}}$ is still required to accelerate the Krylov subspace solver.

In this work, we consider the following strategies for approximating the inverse of $\overline{\boldsymbol{S}}$ for the solution of (4.21):

1. As a simple approach, we can approximate the inverse of $\overline{\boldsymbol{S}}$ using its blockdiagonal part. Let $\overline{\boldsymbol{B}}_{i j}$ denote the block-diagonal part of the near-field partition $(i, j)$, which consists of the self-interactions of the lowest-level clusters. Then the approximation is

$$
\overline{\boldsymbol{S}}^{-1} \approx \overline{\boldsymbol{M}}_{B D}=\left(\overline{\boldsymbol{B}}_{22}-\overline{\boldsymbol{B}}_{21} \cdot\left(\overline{\boldsymbol{B}}_{11}\right)^{-1} \cdot \overline{\boldsymbol{B}}_{12}\right)^{-1} .
$$

2. For normal formulations and JMCFIE, the resulting partitions and the Schur complement are likely to have some degree of diagonal dominance. Therefore, we expect to benefit from the approximation (4.23). On the other hand, CTF partitions are far from being diagonally dominant, and indeed blockdiagonal preconditioners decelerate the convergence rate of iterative solvers for tangential formulations of PEC problems [29]. Thus, for CTF, instead of the approximation in (4.23), we consider the modification formula [32] that expresses the inverse of $\overline{\boldsymbol{S}}$ as

$$
\overline{\boldsymbol{S}}^{-1}=\left(\overline{\boldsymbol{A}}_{22}^{N F}\right)^{-1}+\left(\overline{\boldsymbol{A}}_{22}^{N F}\right)^{-1} \cdot \overline{\boldsymbol{A}}_{21}^{N F} \cdot \overline{\boldsymbol{S}}^{\prime-1} \cdot \overline{\boldsymbol{A}}_{12}^{N F} \cdot\left(\overline{\boldsymbol{A}}_{22}^{N F}\right)^{-1},
$$

where

$$
\overline{\boldsymbol{S}}^{\prime}=\overline{\boldsymbol{A}}_{11}^{N F}-\overline{\boldsymbol{A}}_{12}^{N F} \cdot\left(\overline{\boldsymbol{A}}_{22}^{N F}\right)^{-1} \cdot \overline{\boldsymbol{A}}_{21}^{N F} .
$$

The modification formula is also known as the Woodbury matrix identity [24] or the matrix inversion lemma in control theory [33], or the ShermanMorrison-Woodbury formula in many disciplines, including CEM [27, 28]. To obtain an approximate inverse for $\overline{\boldsymbol{S}}$, we discard the second term in $\overline{\boldsymbol{S}}^{\prime}$ and approximate the inverses of $\overline{\boldsymbol{A}}_{11}^{N F}$ and $\overline{\boldsymbol{A}}_{22}^{N F}$ with SAIs, i.e.,

$$
\begin{aligned}
\overline{\boldsymbol{S}}^{-1} \approx \overline{\boldsymbol{M}}_{M F} & =\overline{\boldsymbol{M}}_{22}+\overline{\boldsymbol{M}}_{22} \cdot \overline{\boldsymbol{A}}_{21}^{N F} \cdot \overline{\boldsymbol{M}}_{11} \cdot \overline{\boldsymbol{A}}_{12} \cdot \overline{\boldsymbol{M}}_{22} \\
& =\overline{\boldsymbol{M}}_{22} \cdot\left(\overline{\boldsymbol{I}}+\overline{\boldsymbol{A}}_{21}^{N F} \cdot \overline{\boldsymbol{M}}_{11} \cdot \overline{\boldsymbol{A}}_{12}^{N F} \cdot \overline{\boldsymbol{M}}_{22}\right),
\end{aligned}
$$

where $\overline{\boldsymbol{M}}_{22}$ denotes the SAI of $\overline{\boldsymbol{A}}_{22}^{N F}$. Note that $\overline{\boldsymbol{A}}_{22}^{N F}=\overline{\boldsymbol{A}}_{11}^{N F}$ for CTF; hence, we need to construct and store only one SAI. The application of (4.27) can be performed by sparse MVMs during the iterative solution of (1.1), without the need to store any matrices other than SAI. 
3. We can approximate the inverse of the Schur complement matrix by

$$
\overline{\boldsymbol{S}}^{-1} \approx\left(\overline{\boldsymbol{A}}_{22}^{N F}\right)^{-1} \approx \overline{\boldsymbol{M}}_{22},
$$

assuming the first term in the RHS of (4.7) is the dominant term in the Schur complement matrix. $\overline{\boldsymbol{M}}_{22}$ denotes the SAI of $\overline{\boldsymbol{A}}_{22}^{N F}$. Again, we need to construct a second SAI only for MNMF.

4. Finally, by employing an incomplete matrix-matrix multiplication, we generate an explicit SAI for $\overline{\boldsymbol{S}}$ that involves both of its first and second terms. First, we compute a sparse approximation to $\bar{S}$ in the form of

$$
\widetilde{\overline{\boldsymbol{S}}}=\overline{\boldsymbol{A}}_{22}^{N F}-\overline{\boldsymbol{A}}_{21}^{N F} \odot \overline{\boldsymbol{M}}_{11} \odot \overline{\boldsymbol{A}}_{12},
$$

where $\odot$ denotes an incomplete matrix-matrix multiplication obtained by retaining the near-field sparsity pattern and $\overline{\boldsymbol{M}}_{11}$ is the SAI of $\overline{\boldsymbol{A}}_{11}^{N F}$. Then the approximation is performed as

$$
\overline{\boldsymbol{S}}^{-1} \approx \widetilde{\overline{\boldsymbol{S}}}^{-1} \approx \overline{\boldsymbol{M}}_{\text {Schur }},
$$

where $\overline{\boldsymbol{M}}_{\text {Schur }}$ denotes an SAI approximation to the inverse of $\widetilde{\overline{\boldsymbol{S}}}$. In our implementation, the block entries of the near-field partitions are stored rowwise. Therefore, the incomplete matrix-matrix multiplication can be performed in $\mathcal{O}(N)$ time using the $i k j$ loop order of the block matrix-matrix multiplication [24] so that the block entries of the matrices are accessed rowwise. Details of this operation are elucidated with a pseudocode in Figure 2. Note that the "if statement" in the innermost loop ensures that a block $\overline{\boldsymbol{C}}_{i j}$ is updated only if clusters $i$ and $j$ are in the near-field zone of each other. In this way, the near-field sparsity pattern is preserved for the product matrix $\bar{C}$.

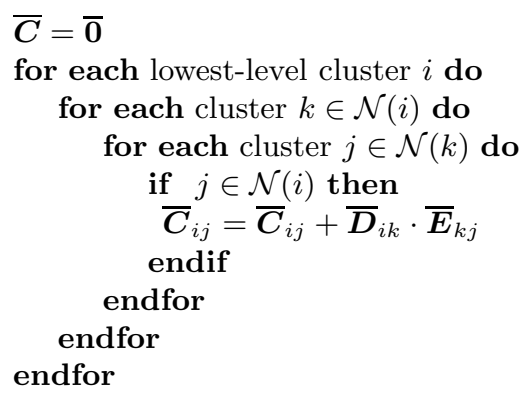

FIG. 2. Incomplete matrix-matrix multiplication of $\overline{\boldsymbol{C}}=\overline{\boldsymbol{D}} \cdot \overline{\boldsymbol{E}}$, where $\overline{\boldsymbol{C}}, \overline{\boldsymbol{D}}$, and $\overline{\boldsymbol{E}}$ are block near-field matrices with the same sparsity pattern. $\overline{\boldsymbol{C}}_{i j}$ denotes the block of the near-field matrix $\overline{\boldsymbol{C}}$ that corresponds to the interaction of cluster $i$ with cluster $j . \mathcal{N}(i)$ denotes the clusters that are in the near-field zone of cluster $i$.

We evaluate the aforementioned approximations in Figures 3, 4, and 5, where we depict the eigenvalues of the preconditioned Schur complement matrices. We summarize our comments as follows:

- In Figure 3, we depict $\overline{\boldsymbol{M}}_{M F} \cdot \overline{\boldsymbol{S}}$ for CTF and $\overline{\boldsymbol{M}}_{B D} \cdot \overline{\boldsymbol{S}}$ for other formulations. We see that the clustering (or localization) of the eigenvalues diminishes with increasing the dielectric constant, particularly for CTF and CNF. 

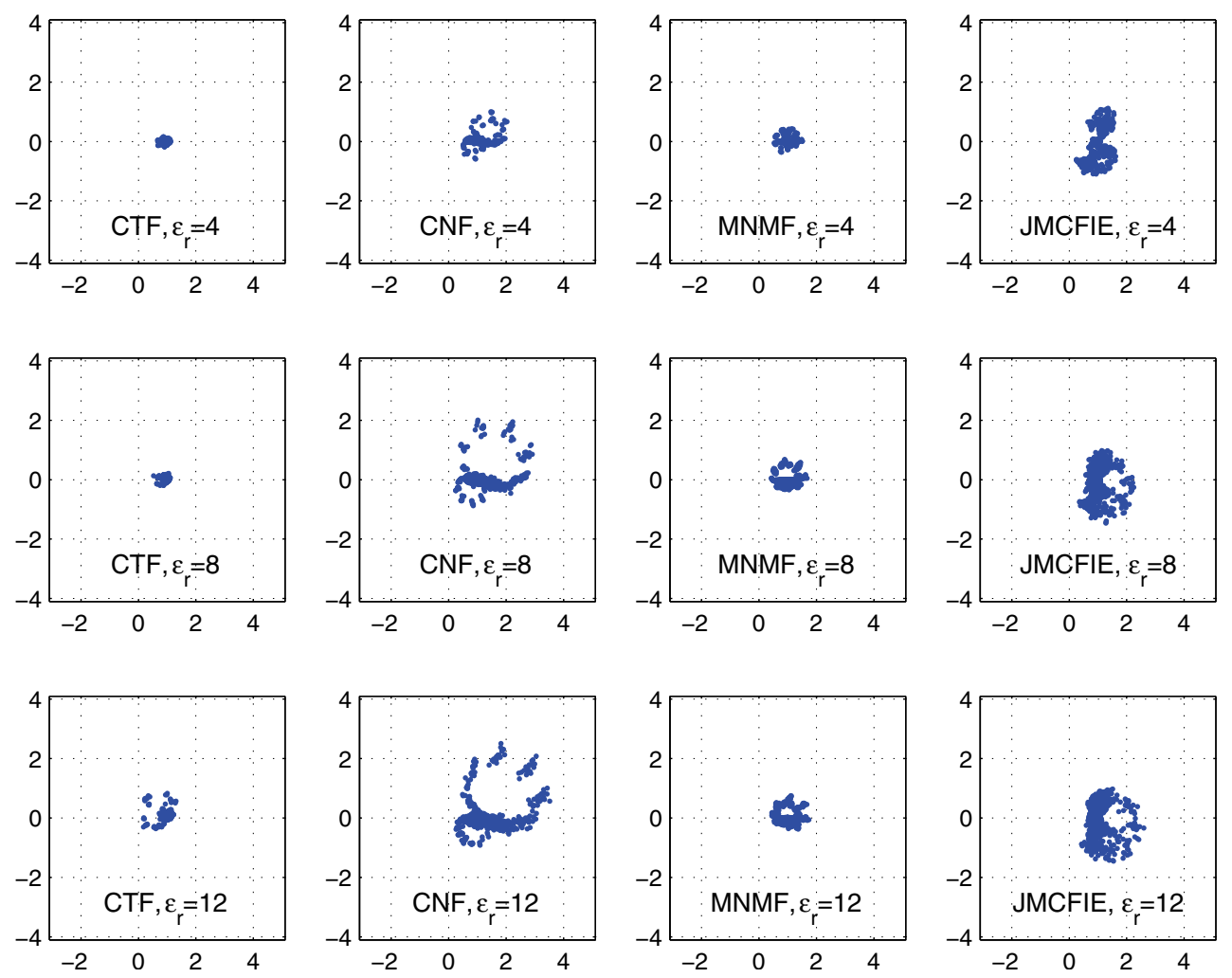

FIG. 3. Eigenvalues of preconditioned Schur complement $\overline{\boldsymbol{S}}$ for increasing dielectric constants of 4,8 , and 12. CTF is preconditioned with $\overline{\boldsymbol{M}}_{M F}$, whereas $\overline{\boldsymbol{M}}_{B D}$ is used as the preconditioner for the other formulations.

Even though the scattering (or spread) of the eigenvalues of CTF with $\overline{\boldsymbol{M}}_{B D}$ is much worse than that of CNF (not shown here), interestingly, the spectra of JMCFIE are less affected from the increase in the dielectric constant than those of CTF and CNF. This can be related to the stronger diagonal dominance of matrices produced with combined formulations than those of tangential formulations [29]. Nonetheless, from the spectra in Figure 3, we conclude that the approximations (4.23) and (4.27) are significantly poorer than (4.22) for all formulations.

- When we omit the second term of the Schur complement matrix in (4.7) and perform the approximation (4.28), we observe from Figure 4 that the spectra of $\mathrm{CNF}$ are extensively scattered with an increasing dielectric constant. Even though not as much as those of CNF, the spectra of CTF are also scattered. JMCFIE, being a combination of $\mathrm{CTF}$ and CNF, is also affected from the scattering of CNF and CTF. Hence, we conclude that this approximation is problematic for high dielectric constants in CTF, CNF, and JMCFIE. MNMF, on the other hand, is less affected from the increase in the dielectric constant. However, when we compare Figures 4 and 1, we conclude that the approximation (4.28) is also significantly poorer than (4.22) for MNMF.

- From Figure 5, it is clear that the best approximation for the Schur complement $\overline{\boldsymbol{S}}$ is provided by $\overline{\boldsymbol{M}}_{\text {Schur }}$. Clusterings of CTF, MNMF, and JMCFIE 

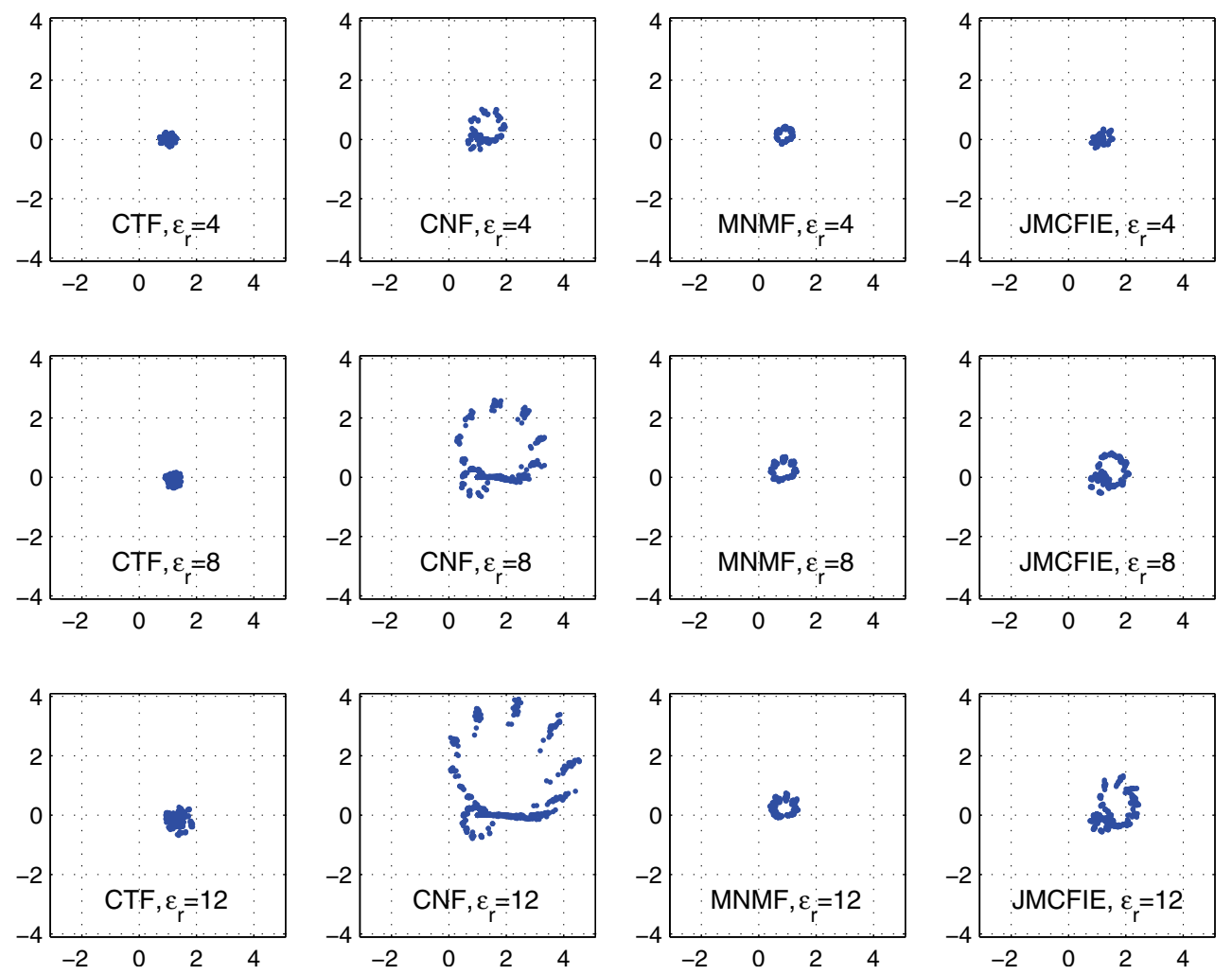

FIG. 4. Eigenvalues of $\overline{\boldsymbol{M}}_{22} \cdot \overline{\boldsymbol{S}}$ for different formulations and increasing dielectric constants of 4,8 , and 12 .

are tight, whereas CNF exhibits slightly looser clustering. When we compare Figures 5 and 1, we observe that the approximation (4.22) is as good as (4.30) for CTF. For other formulations, clusterings in Figure 5 are a little looser compared to those in Figure 1.

From these discussions, we conclude that $\overline{\boldsymbol{M}}_{\text {Schur }}$ provides the most appropriate approximation to the inverse of the Schur complement matrix $\overline{\boldsymbol{S}}$. The other two approximate inverses, $\overline{\boldsymbol{M}}_{B D}$ and $\overline{\boldsymbol{M}}_{22}$, have lower setup and memory costs, but they are far from ensuring the requirement that the approximation for $\overline{\boldsymbol{S}}$ should be as good as that of $\overline{\boldsymbol{A}}_{11}$. On the other hand, in the context of a nested iterative solver (e.g., [31]), $\overline{\boldsymbol{M}}_{\text {Schur }}$ and other approximations, i.e., (4.23), (4.27), and (4.28), can also be utilized as inner preconditioners for iterative solutions of $\overline{\boldsymbol{S}}$, and this will be the subject of another study [44].

5. Numerical results. We use the following setup in our experiments:

- Computations are performed on an Intel Xeon 5355 processor with 16 GB of available memory.

- The generalized minimal residual method (GMRES) [51] with no restart is used as the iterative solver [3]. Even though it is not reported in detail here, contrary to findings in [55], we observe a significant difference between the performances of GMRES and other nonoptimal solvers, such as conjugate gradient squared (CGS) or biconjugate gradient stabilized (BiCGStab). 

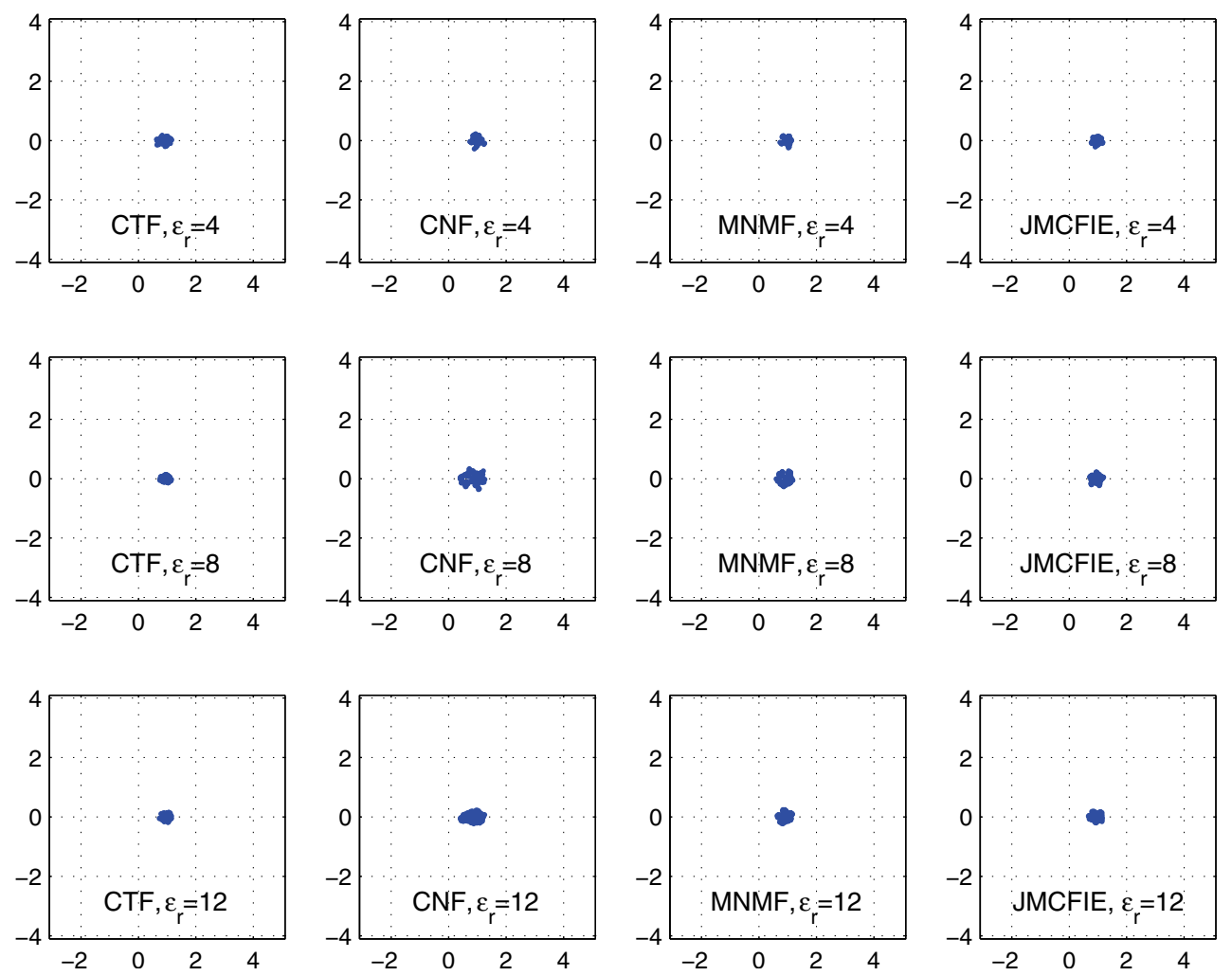

FIG. 5. Eigenvalues of $\overline{\boldsymbol{M}}_{\text {Schur }} \cdot \overline{\boldsymbol{S}}$ for different formulations and increasing dielectric constants of 4,8 , and 12 .

Comparisons of MVM counts for the sphere problem presented in section 5.1 and in [22] demonstrate the superiority of GMRES. We note that the performance difference of GMRES and other nonoptimal solvers is even more severe for the real-life problems of sections 5.2 and 5.3.

- Iterations are performed until the norm of the initial residual is reduced by a factor of $10^{-3}$. This error level is practical [12] and in accordance with the controllable error performed in MLFMA.

- Solutions are started with a zero initial guess and terminated if a maximum of 1,000 iterations is reached.

For comparison purposes, we provide solutions with the no-preconditioner (No PC) case, a four-partition block-diagonal preconditioner (4PBDP) [22], two ILU-type preconditioners, and an SAI preconditioner. SAI and ILU-type preconditioners are applied to the whole matrix, without exploiting the $2 \times 2$ partitioned structure. 4 PBDP is a simple preconditioner constructed by the inclusion of only self-interactions of the lowest-level clusters in each partition. Among several types of ILU preconditioners, the dual-threshold ILUT preconditioner [51] has been shown to be very ineffective in a finite-element implementation of the Navier-Stokes equations [13]. In CEM, however, ILU-type preconditioners have been successfully employed for surface integralequation formulations of PEC problems [42]. For the ILUT preconditioner, we set the threshold values so that it uses up the same amount of memory as ILU(0) and the 
TABLE 1

Salient features of the sphere problems investigated in this study.

\begin{tabular}{|c|c|c|r|c|r|}
\hline \multicolumn{2}{|c|}{ Problem } & $\begin{array}{c}\text { Frequency } \\
(\mathrm{GHz})\end{array}$ & $\begin{array}{c}\text { Size } \\
(\lambda)\end{array}$ & $\begin{array}{c}\text { MLFMA } \\
\text { levels }\end{array}$ & $\begin{array}{c}\text { Number of } \\
\text { unknowns }\end{array}$ \\
\hline \multirow{5}{*}{} & $\mathrm{S} 1$ & 1.0 & 2 & 4 & 7,446 \\
\cline { 2 - 7 } & $\mathrm{S} 2$ & 1.5 & 3 & 5 & 16,728 \\
\cline { 2 - 6 } & $\mathrm{S} 3$ & 3.0 & 6 & 6 & 65,724 \\
\cline { 2 - 6 } & $\mathrm{S} 4$ & 6.0 & 12 & 7 & 264,006 \\
\cline { 2 - 6 } & S5 & 8.5 & 17 & 8 & 540,450 \\
\hline \hline
\end{tabular}

near-field matrix [42]. We also implement an SAI preconditioner for the whole system by using the nonzero pattern of the near-field matrix. Note that the Schur complement preconditioners described in section 4 require only half the memory consumed by the ILU-type and SAI preconditioners.

We first evaluate the proposed preconditioners on a sphere problem, which has an inner dielectric constant of 4.0. The sphere is a widely used geometry in CEM since its analytical solutions are available via Mie-series solutions. We refer to [22] for a comparison of the different integral formulations with respect to accuracy of the solutions. Furthermore, since the sphere geometry is trivially reproducible, it is an important benchmarking problem, providing an opportunity for the evaluation of the performance of the proposed preconditioners with respect to other preconditioners. However, with possible high dielectric constants and complex shapes, real-life problems are more important for judging the quality of a preconditioner. Therefore, we also consider two real-life problems: a lens with a dielectric constant of 12.0 [47] and a photonic crystal with a dielectric constant of 11.56 [37].

5.1. The sphere problem. In Table 1 , we present solution frequencies, diameters in terms of free-space wavelength, number of MLFMA levels, and number of unknowns relating to the sphere problem. We deliberately solve problems with increasing sizes to make a reasonable judgment about the preconditioner, because near-field matrices become sparser as the number of MLFMA levels increases.

5.1.1. Setup times. The setup of the Schur complement preconditioners is composed of the construction of $\overline{\boldsymbol{M}}_{11}$ (SAI of $\overline{\boldsymbol{A}}_{11}^{N F}$ ) and $\overline{\boldsymbol{M}}_{\text {Schur }}$ (SAI of the approximate Schur complement matrix $\overline{\boldsymbol{S}}$ ). In Table 2 , we compare these setup times with those of ILUTP (ILUT with 0.5 pivoting tolerance), ILU(0), and SAI. The setup of 4PPBDP is negligible.

From Table 2, we see that setup times of ILUTP and SAI are much larger than those of the others, particularly for the S5 problem. The time required for the setup of ILU(0) is six to eight times less than that of the Schur complement preconditioners, which require the constructions of both $\overline{\boldsymbol{M}}_{11}$ and $\overline{\boldsymbol{M}}_{\text {Schur }}$. As the following tables will reveal, however, both of these times are insignificant compared to the iterative solution times of the problems. Finally, note that the setup time of $\overline{\boldsymbol{M}}_{\text {Schur }}$ is only slightly higher than that of $\overline{\boldsymbol{M}}_{11}$ because of the efficiently implemented incomplete matrix-matrix multiplication described in Figure 2.

5.1.2. ILU-type, SAI, and simple preconditioners. For CTF, similar to the results of [13], we observe that the ILU-type preconditioners have an instability issue. In particular, with ILU(0), the condition estimates [14] turn out to be very 
TABLE 2

Setup times (in minutes) of ILU-type preconditioners and SAIs of $\overline{\boldsymbol{A}}_{11}^{N F}$ and the Schur complement matrix $\overline{\boldsymbol{S}}$.

\begin{tabular}{|c|r|c|c|c|c|}
\hline Problem & ILUTP & ILU(0) & \multicolumn{1}{c|}{ SAI } & $\overline{\boldsymbol{M}}_{11}$ & $\overline{\boldsymbol{M}}_{\text {Schur }}$ \\
\hline S1 & 0.16 & 0.02 & 0.59 & 0.08 & 0.08 \\
\hline S2 & 0.56 & 0.05 & 1.38 & 0.18 & 0.19 \\
\hline S3 & 3.84 & 0.19 & 5.40 & 0.68 & 0.73 \\
\hline S4 & 20.54 & 0.77 & 22.54 & 2.82 & 3.05 \\
\hline S5 & 158.21 & 2.17 & 45.98 & 5.75 & 6.23 \\
\hline
\end{tabular}

high for some large sphere problems. The same situation also arises for ILUT, but the instability can be removed in this case if pivoting with 0.5 tolerance is applied. Other formulations that are of the second-kind do not exhibit any instability and ILU(0) performs the best among the ILU-type preconditioners for those formulations. Therefore, we employ ILU(0) for formulations other than CTF, and ILUTP for CTF. Our comments on the results of No PC, 4PBDP, SAI, and ILU-type preconditioners presented in Table 3 are as follows:

- For all formulations, the no-restart GMRES solves all sphere problems successfully. However, the number of iterations is very high in some instances, such as the CNF solution of S4. Moreover, some large instances of these problems cannot be solved with other nonoptimal solvers. For example, the solutions of S5 do not converge with BiCGStab for CNF, MNMF, and JMCFIE.

- In accordance with the findings in [22], we observe that 4PBDP worsens the convergence behavior of CTF. In that paper, it is shown that for other formulations, CGS and BiCGStab solutions of the sphere geometry can significantly be improved with 4PBDP. Nevertheless, 4PBDP is, in general, less effective on the convergence of large problems when GMRES is employed as the iterative solver.

- Considering the solutions with CTF, ILUTP provides a significant improvement over No PC only for the S3 case. Solutions with CNF, on the other hand, significantly benefit from ILU(0). For better-conditioned JMCFIE and MNMF, ILU(0) provides minor improvements over 4PBDP.

- For CTF and MNMF, the SAI preconditioner for the whole system worsens the convergence rate. This is also true for the largest two solutions of JMCFIE. For CNF, SAI provides minor improvements with respect to much cheaper 4PBDP. We note that failure or limited success of standard algebraic preconditioners that do not take into account the partitioned structure, such as ILU-type or SAI, has also been reported previously [6, 13].

5.1.3. Schur complement preconditioners. In Table 4, we present iteration counts and total solution times of Schur complement preconditioners. We first note that per-iteration times of all Schur complement preconditioners are very close to each other. Even though the application of DASP requires two, the applications of UTASP and LTASP require three, and the application of ASP requires four multiplications with $N \times N$ sparse partitions, the time required for these multiplications is much less than the time required for the far-field computations performed by MLFMA. Furthermore, the complexity of near-field partition is $\mathcal{O}(N)$, whereas MLFMA scales with $\mathcal{O}(N \log N)$. As a result, per-iteration times are dominated by the MLFMA 
TABLE 3

Performances of the $4 P B D P$, ILU-type, and SAI preconditioners and No PC on the sphere problem.

\begin{tabular}{|c|c|c|c|c|c|c|c|c|}
\hline \multirow[b]{3}{*}{ Problem } & \multicolumn{8}{|c|}{ CTF } \\
\hline & \multicolumn{2}{|c|}{ No PC } & \multicolumn{2}{|c|}{$4 \mathrm{PBDP}$} & \multicolumn{2}{|c|}{ ILUTP } & \multicolumn{2}{|c|}{ SAI } \\
\hline & iter & time & iter & time & iter & time & iter & time \\
\hline S1 & 179 & 7 & 467 & 18 & 149 & 6 & 216 & 9 \\
\hline S2 & 167 & 21 & 668 & 85 & 138 & 19 & 291 & 38 \\
\hline S3 & 471 & 313 & $t$ & - & 284 & 198 & 472 & 319 \\
\hline $\mathrm{S} 4$ & 291 & 912 & $\dagger$ & - & 268 & 851 & 732 & 2,327 \\
\hline \multirow[t]{4}{*}{$\mathrm{S} 5$} & 271 & 2,028 & $\dagger$ & - & 273 & 2,198 & 908 & 6,826 \\
\hline & \multicolumn{8}{|c|}{ CNF } \\
\hline & \multicolumn{2}{|c|}{ No PC } & \multicolumn{2}{|c|}{ 4PBDP } & \multicolumn{2}{|c|}{$\overline{I L U}(0)$} & \multicolumn{2}{|c|}{ SAI } \\
\hline & iter & time & iter & time & iter & time & iter & time \\
\hline S1 & 67 & 3 & 45 & 2 & 27 & 1 & 46 & 2 \\
\hline $\mathrm{S} 2$ & 140 & 18 & 89 & 11 & 46 & 6 & 79 & 11 \\
\hline S3 & 171 & 113 & 126 & 83 & 61 & 41 & 96 & 69 \\
\hline $\mathrm{S} 4$ & 968 & 3,065 & 516 & 1,894 & 161 & 515 & 318 & 1,031 \\
\hline \multirow[t]{4}{*}{ S5 } & 390 & 2,916 & 386 & 2,880 & 120 & 902 & 315 & 2,413 \\
\hline & \multicolumn{8}{|c|}{ MNMF } \\
\hline & \multicolumn{2}{|c|}{ No PC } & \multicolumn{2}{|c|}{ 4PBDP } & \multicolumn{2}{|c|}{$\operatorname{ILU}(0)$} & \multicolumn{2}{|c|}{$\overline{\text { SAI }}$} \\
\hline & iter & time & iter & time & iter & time & iter & time \\
\hline $\mathrm{S} 1$ & 47 & 2 & 32 & 1 & 27 & 1 & 225 & 9 \\
\hline S2 & 71 & 9 & 51 & 7 & 39 & 5 & 151 & 21 \\
\hline S3 & 112 & 73 & 85 & 56 & 63 & 42 & 224 & 155 \\
\hline $\mathrm{S} 4$ & 192 & 605 & 161 & 504 & 116 & 368 & 559 & 1,792 \\
\hline \multirow[t]{4}{*}{ S5 } & 187 & 1,405 & 165 & 1,240 & 108 & 826 & & \\
\hline & \multicolumn{8}{|c|}{ JMCFIE } \\
\hline & \multicolumn{2}{|c|}{ No PC } & \multicolumn{2}{|c|}{$4 \mathrm{PBDP}$} & \multicolumn{2}{|c|}{ ILU(0) } & \multicolumn{2}{|c|}{ SAI } \\
\hline & iter & time & iter & time & iter & time & iter & time \\
\hline S1 & 79 & 3 & 53 & 2 & 31 & 1 & 61 & 3 \\
\hline S2 & 93 & 12 & 62 & 8 & 36 & 5 & 91 & 13 \\
\hline S3 & 139 & 92 & 100 & 67 & 68 & 46 & 91 & 66 \\
\hline S4 & 223 & 706 & 141 & 444 & 102 & 326 & 232 & 762 \\
\hline S5 & 143 & 1,075 & 111 & 836 & 102 & 805 & 160 & 1,259 \\
\hline
\end{tabular}

operations and iteration times are in accordance with the iteration counts. For a certain formulation, when we can decide that some of the preconditioners behave worse than the others, we omit them for the largest S5 problem. For example, we omit UTASP and LTASP solutions of S5 for CTF.

Our comments on the results presented in Table 4, also compared to those in Table 3, are as follows:

- ASP is the best-performing preconditioner among the Schur complement preconditioners except for the $\mathrm{S} 4$ solution of $\mathrm{CNF}$ and the largest three problems of MNMF; it is possible that the indefiniteness of the matrices causes this [17]. While improving the preconditioner, the eigenvalues with a negative real part move progressively towards the point $(1,0)$. Meanwhile, however, some eigenvalues may be very close to zero, slowing down the convergence. 
TABLE 4

Performances of the Schur complement preconditioners on the sphere problem.

\begin{tabular}{|c|c|c|c|c|c|c|c|c|c|c|c|c|}
\hline \multirow{3}{*}{$\begin{array}{c}\text { Prob- } \\
\text { lem }\end{array}$} & \multicolumn{6}{|c|}{ CTF } & \multicolumn{6}{|c|}{ CNF } \\
\hline & \multicolumn{2}{|c|}{ UTASP } & \multicolumn{2}{|c|}{ LTASP } & \multicolumn{2}{|c|}{$\mathrm{ASP}$} & \multicolumn{2}{|c|}{ UTASP } & \multicolumn{2}{|c|}{ LTASP } & \multicolumn{2}{|c|}{$\mathrm{ASP}$} \\
\hline & iter & time & iter & time & iter & time & iter & time & iter & time & iter & time \\
\hline S1 & 53 & 2.2 & 48 & 2.0 & 43 & 1.8 & 33 & 1.4 & 30 & 1.3 & 27 & 1.2 \\
\hline S2 & 60 & 7.9 & 55 & 7.3 & 47 & 6.3 & 57 & 7.7 & 57 & 7.7 & 46 & 6.2 \\
\hline S3 & 147 & 98.7 & 121 & 81.5 & 103 & 69.8 & 64 & 43.8 & 59 & 40.5 & 55 & 38.0 \\
\hline $\mathrm{S} 4$ & 209 & 664.4 & 178 & 566.7 & 144 & 459.2 & 130 & 416.7 & 204 & 651.7 & 158 & 506.9 \\
\hline \multirow[t]{4}{*}{ S5 } & \multicolumn{2}{|r|}{ 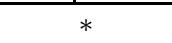 } & \multicolumn{2}{|r|}{ 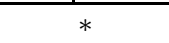 } & 147 & $1,109.7$ & 97 & 747.8 & & 97 & 741.0 \\
\hline & \multicolumn{6}{|c|}{ MNMF } & \multicolumn{6}{|c|}{ JMCFIE } \\
\hline & \multicolumn{2}{|c|}{ UTASP } & \multicolumn{2}{|c|}{ LTASP } & \multicolumn{2}{|c|}{ ASP } & \multicolumn{2}{|c|}{ UTASP } & \multicolumn{2}{|c|}{ LTASP } & \multicolumn{2}{|c|}{ ASP } \\
\hline & iter & time & iter & time & iter & time & iter & time & iter & time & iter & time \\
\hline S1 & 45 & 1.9 & 33 & 1.4 & 29 & 1.3 & 33 & 1.4 & 35 & 1.5 & 29 & 1.3 \\
\hline S2 & 66 & 8.7 & 43 & 5.8 & 42 & 5.7 & 40 & 5.5 & 40 & 5.5 & 34 & 4.7 \\
\hline S3 & 123 & 83.3 & 67 & 46.0 & 70 & 48.0 & 63 & 43.4 & 76 & 52.1 & 55 & 38.1 \\
\hline $\mathrm{S} 4$ & 235 & 752.0 & 122 & 391.9 & 132 & 423.8 & 93 & 301.7 & 124 & 400.9 & 84 & 273.6 \\
\hline $\mathrm{S} 5$ & & * & 103 & 788.7 & 128 & 982.3 & & $*$ & & 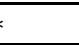 & 77 & 595.7 \\
\hline
\end{tabular}

Notes: "iter" and "time" denote the number of iterations and total solution time in minutes. An asterisk "*" denotes that the problem is not solved with that particular preconditioner.

- For CTF, ASP reduces solution times of the sphere problems by a factor of two to four, compared to ILUTP and No PC. For CNF, ASP provides a reduction by a factor of three to six with respect to $4 \mathrm{PBDP}$. ILU(0) solves CNF systems as fast as ASP, but for S5, solutions with ASP converge faster. JMCFIE solutions are also obtained about two times faster with ASP than with 4PBDP. ILU(0) is better than 4PBDP for JMCFIE, but it is worse than ASP. Finally, MNMF benefits the least from the Schur complement preconditioners. Nonetheless, for large problems, LTASP provides an approximate $30 \%$ reduction in time compared to 4PBDP. ILU(0) solves MNMF problems as fast as the Schur complement preconditioners do.

- When we compare the formulations considering their performances with ASP, we observe that JMCFIE systems are solved with the lowest iteration counts and CTF systems are solved with the highest iteration counts. Although the iteration counts of MNMF are much less than those of CNF without a preconditioner, $\mathrm{CNF}$ benefits more from preconditioning. As a result, iteration counts of these formulations become close to each other when an ILU-type or a Schur complement preconditioner is employed.

5.2. The lens problem. For radiometric remote sensing applications, delicate simulations of dielectric lenses are required for a wide spectrum, beginning from 30 $\mathrm{GHz}[47]$. This application gives rise to large problems that are difficult to solve without preconditioning. In this section, we analyze preconditioned iterative solutions of this important problem. We increase the frequency by $30 \mathrm{GHz}$ intervals, up to 120 GHz. The resulting problems are listed in Table 5. The lens problem involves a dielectric half sphere with a high dielectric constant of 12.0.

5.2.1. ILU-type, SAI, and simple preconditioners. CTF solutions of lens problems do not suffer from the instability of ILU-type preconditioners. ILU(0) performs better than ILUT and ILUTP for all formulations. Hence, for all formulations, 
TABLE 5

Salient features of the lens problems investigated in this study.

\begin{tabular}{|c|c|c|c|c|r|}
\hline \multicolumn{2}{|c|}{ Problem } & $\begin{array}{c}\text { Frequency } \\
(\mathrm{GHz})\end{array}$ & $\begin{array}{c}\text { Size } \\
(\lambda)\end{array}$ & $\begin{array}{c}\text { MLFMA } \\
\text { levels }\end{array}$ & $\begin{array}{c}\text { Number of } \\
\text { unknowns }\end{array}$ \\
\hline \multirow{4}{*}{} & L1 & 30 & 2.5 & 6 & 38,466 \\
\cline { 2 - 6 } & L2 & 60 & 5.0 & 7 & 158,286 \\
\cline { 2 - 6 } & L3 & 90 & 7.5 & 7 & 353,646 \\
\cline { 2 - 5 } & L4 & 120 & 10.0 & 8 & 632,172 \\
\hline
\end{tabular}

we compare ILU(0) with No PC and 4PBDP in Table 6. CNF solutions of L4 with No PC and 4PBDP cannot be completed since the memory requirement cannot be met with the available memory after 500 GMRES iterations. This is also the case for the SAI solution of L4 with CTF. Our comments on the results are as follows:

- We observe that CNF cannot solve L2, L3, and L4 problems without a preconditioner. CTF and JMCFIE converge with similar rates and MNMF converges the fastest. These results are in accordance with the discussion in section 2.5. A high dielectric constant degrades the conditioning of normal formulations [62]. In addition, the spectra illustrated in Figures 3, 4, and 5 reveal that $\mathrm{CNF}$ is negatively affected more than the others by an increase in the dielectric constant. As a combination of CTF and CNF, JMCFIE is also adversely affected by a high dielectric constant. Consequently, its iteration counts turn out to be close to those of CTF for the lens problem.

- JMCFIE benefits more from 4PBDP than MNMF, and iteration counts for these formulations become close to each other with 4 PBDP. For the largest problem L4, JMCFIE converges even faster than MNMF. Superiority of JMCFIE over MNMF for large problems has also been demonstrated in [22].

- ILU(0) performs significantly better than 4PBDP on the lens problem. All of the formulations can be solved faster with ILU(0), but second-kind formulations are accelerated more than CTF since they have more diagonally dominant matrices than CTF does.

- Similar to the sphere problem, SAI decelerates the convergence rate of CTF and MNMF. For CNF and JMCFIE formulations, it performs better than 4PBDP, but poorer than ILU(0).

5.2.2. Schur complement preconditioners. In Table 7, we present solutions of the lens problems with the Schur complement preconditioners. Solutions of L1 and L2 show that ASP performs significantly better than other Schur complement preconditioners; hence, we perform solutions of the larger L3 and L4 problems only with ASP. We summarize our comments on the results as follows:

- With ASP, all of the formulations can be solved much faster than with No PC or 4PBDP, and solution times are reduced twofold to fivefold, depending on the type of formulation. The number of iterations for JMCFIE and MNMF are close to each other, and are approximately half of the number of iterations for CTF and CNF.

- For CTF and JMCFIE, ASP performs significantly better than ILU(0). But for CNF and MNMF, ILU(0) performs slightly better than ASP.

5.3. The photonic crystal problem. We conclude this section with a comparative investigation of the performance of ASP on a complicated structure, namely, 
TABLE 6

Performances of the $4 P B D P, I L U(0)$, and SAI preconditioners and No PC on the lens problem.

\begin{tabular}{|c|c|c|c|c|c|c|c|c|}
\hline \multirow[b]{3}{*}{ Problem } & \multicolumn{8}{|c|}{ CTF } \\
\hline & \multicolumn{2}{|c|}{ No PC } & \multicolumn{2}{|c|}{$4 \mathrm{PBDP}$} & \multicolumn{2}{|c|}{ ILU(0) } & \multicolumn{2}{|c|}{ SAI } \\
\hline & iter & time & iter & time & iter & time & iter & time \\
\hline L1 & 205 & 162 & \multicolumn{2}{|c|}{$*$} & 105 & 61 & 272 & 161 \\
\hline L2 & 278 & 939 & \multicolumn{2}{|c|}{ * } & 152 & 442 & 541 & 1,575 \\
\hline L3 & 276 & 1,853 & \multicolumn{2}{|c|}{ * } & 227 & 1,525 & 465 & 3,137 \\
\hline \multirow[t]{4}{*}{$\mathrm{L} 4$} & 321 & 4,458 & \multicolumn{2}{|c|}{$*$} & 229 & 3,165 & \multicolumn{2}{|c|}{ MLE } \\
\hline & \multicolumn{8}{|c|}{ CNF } \\
\hline & \multicolumn{2}{|c|}{ No PC } & \multicolumn{2}{|c|}{ 4PBDP } & \multicolumn{2}{|c|}{ ILU(0) } & \multicolumn{2}{|c|}{ SAI } \\
\hline & iter & time & iter & time & iter & time & iter & time \\
\hline L1 & 368 & 292 & 140 & 102 & 44 & 26 & 92 & 57 \\
\hline L2 & $\dagger$ & - & 333 & 1,115 & 87 & 257 & 145 & 428 \\
\hline L3 & $\dagger$ & - & 406 & 2,734 & 87 & 589 & 198 & 1,352 \\
\hline \multirow[t]{4}{*}{$\mathrm{L} 4$} & \multicolumn{2}{|c|}{ MLE } & \multicolumn{2}{|c|}{ MLE } & 117 & 1,627 & 263 & 3,694 \\
\hline & \multicolumn{8}{|c|}{ MNMF } \\
\hline & \multicolumn{2}{|c|}{ No PC } & \multicolumn{2}{|c|}{$4 \mathrm{PBDP}$} & \multicolumn{2}{|c|}{ ILU(0) } & \multicolumn{2}{|c|}{ SAI } \\
\hline & iter & time & iter & time & iter & time & iter & time \\
\hline L1 & 78 & 51 & 52 & 34 & 32 & 19 & $\dagger$ & - \\
\hline $\mathrm{L} 2$ & 114 & 386 & 86 & 288 & 43 & 124 & $\dagger$ & - \\
\hline L3 & 146 & 970 & 131 & 871 & 48 & 328 & $\dagger$ & - \\
\hline \multirow[t]{4}{*}{ L4 } & 166 & 2,282 & 166 & 2,284 & 52 & 720 & $\dagger$ & - \\
\hline & \multicolumn{8}{|c|}{ JMCFIE } \\
\hline & \multicolumn{2}{|c|}{ No PC } & \multicolumn{2}{|c|}{ 4PBDP } & \multicolumn{2}{|c|}{ ILU(0) } & \multicolumn{2}{|c|}{ SAI } \\
\hline & iter & time & iter & time & iter & time & iter & time \\
\hline L1 & 138 & 110 & 77 & 57 & 40 & 23 & 70 & 44 \\
\hline L2 & 227 & 786 & 114 & 391 & 67 & 198 & 87 & 266 \\
\hline L3 & 276 & 1,850 & 128 & 860 & 71 & 501 & 110 & 766 \\
\hline L4 & 310 & 4,310 & 135 & 1,872 & 88 & 1,224 & 122 & 1,745 \\
\hline
\end{tabular}

Notes: "iter" and "time" denote the number of iterations and total solution time in minutes. An asterisk "*" denotes that the problem is not solved with that particular preconditioner. A dagger " + " denotes nonconvergence. "MLE" denotes that memory limitation is exceeded.

a photonic crystal waveguide, which is composed of a dielectric slab etched with a waveguiding pattern of holes [37]. An example of the problem and its near-field pattern are shown in Figure 6. The operating frequency is chosen as $8.25 \mathrm{GHz}$. We increase the problem size by enlarging the size of the structure and including more holes, as shown in Table 8. Diameters of the holes are on the order of $0.1 \lambda$; hence, this problem requires a fine meshing of about $0.05 \lambda$ in order to model these small details. As a result, the lowest-level clusters of MLFMA contain more basis functions and the resulting near-field matrices become denser compared to previous problems.

In Table 9, we present information regarding the solutions of the photonic crystal problems with No PC, 4PBDP, ILU(0), and ASP. For CTF solutions, ILU(0) is again more successful than ILUTP and SAI in reducing the iteration counts. The solutions with CNF and MMNMF are omitted for this particular problem because they perform much poorer than CTF and JMCFIE do. We summarize our comments on the results as follows:

- Note that the performance of No PC and that of 4PBDP are worse for this 
TABLE 7

Performances of the Schur complement preconditioners on the lens problem.

\begin{tabular}{|c|c|c|c|c|c|c|c|c|c|c|c|c|}
\hline \multirow{3}{*}{$\begin{array}{c}\text { Prob- } \\
\text { lem }\end{array}$} & \multicolumn{6}{|c|}{ CTF } & \multicolumn{6}{|c|}{ CNF } \\
\hline & \multicolumn{2}{|c|}{ UTASP } & \multicolumn{2}{|c|}{ LTASP } & \multicolumn{2}{|c|}{$\overline{\mathrm{ASP}}$} & \multicolumn{2}{|c|}{ UTASP } & \multicolumn{2}{|c|}{ LTASP } & \multicolumn{2}{|c|}{ ASP } \\
\hline & iter & time & iter & time & iter & time & iter & time & iter & time & iter & time \\
\hline L1 & 129 & 75 & 93 & 55 & 57 & 34 & 101 & 59 & 70 & 41 & 45 & 27 \\
\hline $\mathrm{L} 2$ & 189 & 550 & 135 & 394 & 85 & 249 & 227 & 655 & 160 & 461 & 92 & 266 \\
\hline L3 & \multicolumn{2}{|c|}{ * } & \multicolumn{2}{|c|}{$*$} & $\overline{99}$ & 669 & \multicolumn{2}{|c|}{$*$} & \multicolumn{2}{|c|}{ * } & 94 & 635 \\
\hline \multirow[t]{4}{*}{$\mathrm{L} 4$} & \multicolumn{2}{|c|}{ * } & \multicolumn{2}{|c|}{ * } & 114 & 1,592 & \multicolumn{2}{|c|}{ * } & \multicolumn{2}{|c|}{$*$} & 128 & 1,785 \\
\hline & \multicolumn{6}{|c|}{ MNMF } & \multicolumn{6}{|c|}{ JMCFIE } \\
\hline & \multicolumn{2}{|c|}{ UTASP } & \multicolumn{2}{|c|}{ LTASP } & \multicolumn{2}{|c|}{$\overline{\mathrm{ASP}}$} & \multicolumn{2}{|c|}{ UTASP } & \multicolumn{2}{|c|}{ LTASP } & \multicolumn{2}{|c|}{ ASP } \\
\hline & iter & time & iter & time & iter & time & iter & time & iter & time & iter & time \\
\hline L1 & 90 & 53 & 41 & 25 & 35 & 21 & 56 & 34 & 48 & 29 & 31 & 19 \\
\hline $\mathrm{L} 2$ & 133 & 386 & 54 & 159 & 48 & 142 & 100 & 292 & 80 & 235 & 52 & 154 \\
\hline L3 & \multicolumn{2}{|c|}{ * } & \multicolumn{2}{|c|}{$*$} & 54 & 369 & \multicolumn{2}{|c|}{ * } & \multicolumn{2}{|c|}{$*$} & 54 & 368 \\
\hline $\mathrm{L} 4$ & \multicolumn{2}{|c|}{ * } & \multicolumn{2}{|c|}{$*$} & 60 & 846 & \multicolumn{2}{|r|}{ * } & \multicolumn{2}{|c|}{ * } & 64 & 901 \\
\hline
\end{tabular}

Notes: "iter" and "time" denote the number of iterations and total solution time in minutes. An asterisk " $*$ " denotes that the problem is not solved with that particular preconditioner.

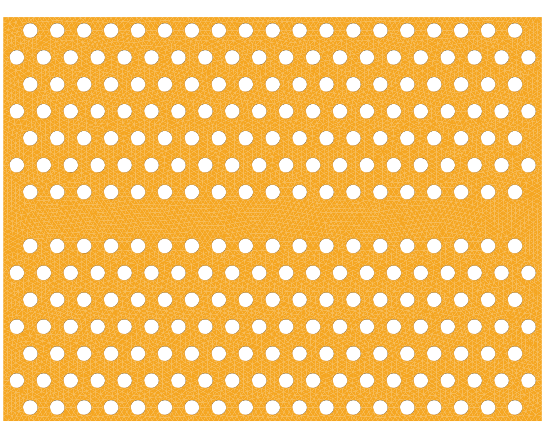

(a)

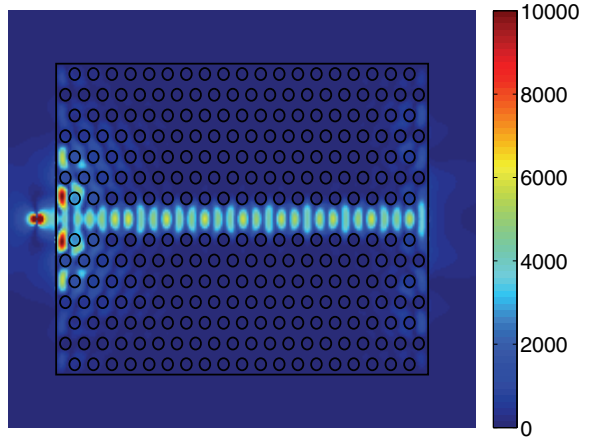

(b)

FIG. 6. (a) A perforated photonic crystal waveguide. (b) Near-zone magnetic fields of the problem when illuminated by a Hertzian dipole.

TABLE 8

Salient features of the photonic crystal problems investigated in this study.

\begin{tabular}{|c|c|c|c|c|r|}
\hline \multicolumn{2}{|c|}{} & $\begin{array}{c}\text { Frequency } \\
(\mathrm{GHz})\end{array}$ & $\begin{array}{c}\text { Number of } \\
\text { holes }\end{array}$ & $\begin{array}{c}\text { MLFMA } \\
\text { levels }\end{array}$ & $\begin{array}{c}\text { Number of } \\
\text { unknowns }\end{array}$ \\
\hline \multirow{3}{*}{ Problem } & PhC1 & \multirow{3}{*}{8.25} & $5 \times 5$ & 4 & 14,226 \\
\cline { 2 - 4 } \cline { 4 - 6 } & & $5 \times 10$ & 5 & 27,798 \\
\cline { 2 - 4 } & PhC2 & $10 \times 15$ & 6 & 162,420 \\
\hline
\end{tabular}

problem than for the sphere and lens problems. Specifically, the largest problem, PhC3, cannot be solved without a preconditioner or with 4PBDP when formulated with CTF. In addition, the iteration counts with JMCFIE for PhC3 turn out to be very high.

- Since the near-field matrix for this problem is significantly denser than for previous problems, ILU(0) performs remarkably well, particularly for JMCFIE. On the other hand, in a double-precision implementation, ILU(0) requires 8.3 
TABLE 9

Comparison of ASP with ILU-type and simple preconditioners for the photonic crystal problem.

\begin{tabular}{|c|c|c|c|c|c|c|c|c|c|c|c|}
\hline \multirow{3}{*}{$\begin{array}{c}\text { Prob- } \\
\text { lem }\end{array}$} & \multicolumn{11}{|c|}{ CTF } \\
\hline & \multicolumn{2}{|c|}{ No PC } & \multicolumn{2}{|c|}{$4 \mathrm{PBDP}$} & \multicolumn{3}{|c|}{$\overline{\mathrm{ILU}(0)}$} & \multicolumn{2}{|c|}{ ASP Setup } & \multicolumn{2}{|c|}{ ASP } \\
\hline & iter & time & iter & time & setup & iter & time & $\overline{\boldsymbol{M}}_{11}$ & $\overline{\bar{M}}_{\text {Schur }}$ & iter & time \\
\hline $\mathrm{PhC1}$ & 397 & 10 & 533 & 14 & 2 & 63 & 2 & 2 & 2 & 37 & 6 \\
\hline $\mathrm{PhC2}$ & 695 & 43 & 881 & 56 & 5 & 141 & 10 & 6 & 6 & 58 & 16 \\
\hline \multirow[t]{4}{*}{ PhC3 } & $\dagger$ & - & $\dagger$ & - & \multicolumn{3}{|c|}{ MLE } & 54 & 57 & 217 & 214 \\
\hline & \multicolumn{11}{|c|}{ JMCFIE } \\
\hline & \multicolumn{2}{|c|}{ No PC } & \multicolumn{2}{|c|}{ 4PBDP } & \multicolumn{3}{|c|}{$\overline{\mathrm{ILU}(0)}$} & \multicolumn{2}{|c|}{ ASP Setup } & \multicolumn{2}{|c|}{ ASP } \\
\hline & iter & time & iter & time & setup & iter & time & $\overline{\boldsymbol{M}}_{11}$ & $\overline{\bar{M}}_{\text {Schur }}$ & iter & time \\
\hline $\mathrm{PhC1}$ & 196 & 6 & 118 & 3 & 2 & 19 & 1 & 2 & 2 & 39 & 6 \\
\hline $\mathrm{PhC} 2$ & 316 & 21 & 183 & 12 & 5 & 32 & 2 & 6 & 6 & 76 & $\overline{18}$ \\
\hline PhC3 & 874 & 374 & 593 & 255 & \multicolumn{3}{|c|}{ MLE } & 54 & 57 & 278 & 248 \\
\hline
\end{tabular}

Notes: "iter" denotes number of iterations. "time" denotes total solution time and "setup" denotes setup time of the preconditioner, in minutes. A dagger " $\dagger$ " denotes nonconvergence. "MLE" denotes that memory limitation is exceeded.

GB of memory for $\mathrm{PhC} 3$, and as a result, this problem does not fit into the available memory.

- We have been able to solve the largest problem (PhC3) only by using ASP. When we compare CTF and JMCFIE solutions with ASP, we observe that $\mathrm{CTF}$ requires fewer number of iterations compared to JMCFIE. Also, CTF uses less memory than JMCFIE, as explained in section 2.5. Moreover, this problem involves a high dielectric constant and many surface singularities. Therefore, we can expect CTF to produce more accurate results than JMCFIE.

6. Conclusion. In the context of surface integral-equation methods for dielectric problems, simultaneous discretization of the surface currents and integral equations leads to matrix equations with $2 \times 2$ partitions. These partitions show some resemblance to the matrices that are obtained in PEC problems. Based on our prior experience with the preconditioning of PEC problems, we have developed robust Schur complement preconditioners for dielectric problems by using the $2 \times 2$ partitioned structure of matrices. Inspired by its success in PEC problems [43], the SAI preconditioner is applied to the $(1,1)$ partition. For the Schur complement, we discuss several approximation strategies and show that obtaining an approximation via sparse matrix-matrix multiplications yields the best results.

To the best of our knowledge, the following conclusions drawn from the numerical experiments are novel and have the potential to change the common wisdom regarding the solutions of surface integral equations for dielectric problems:

- The no-restart GMRES solver is much more robust and efficient for preconditioned and unpreconditioned matrix systems than other nonoptimal solvers.

- When high accuracy is a concern, CTF solutions can be obtained without difficulty by using the Schur complement preconditioners. The lack of diagonal dominance in CTF prevents the success of block-diagonal-type (i.e., 4PBDP [22]), ILU(0), or SAI preconditioners. Although they are known as the most general and effective preconditioners for nonsymmetric and indefinite systems [51], ILUT and ILUTP also have discouraging performances on CTF. 
- Normal formulations and JMCFIE are second-kind integral equations that are expected to yield well-conditioned linear systems. Particularly for large problem sizes, however, effective preconditioning becomes indispensable for these formulations when the problem involves a high dielectric constant. We also note that standard algebraic preconditioners that do not take into account the partitioned structure can perform poorly on such systems.

- Furthermore, the photonic crystal problem shows that the complexity of the geometry and the high dielectric constant may render linear systems obtained from normal formulations unsolvable even with effective preconditioners. Linear systems obtained from JMCFIE can be solved with simple preconditioners, but they require many iterations. When ASP is used, on the other hand, the most accurate CTF solutions can be attained with the lowest iteration counts.

\section{REFERENCES}

[1] G. Alléon, M. Benzi, And L. Giraud, Sparse approximate inverse preconditioning for dense linear systems arising in computational electromagnetics, Numer. Algorithms, 16 (1997), pp. $1-15$.

[2] Z.-Z. BAI, Structured preconditioners for nonsingular matrices of block two-by-two structures, Math. Comp., 75 (2006), pp. 791-815.

[3] S. Balay, W. D. Gropp, L. C. McInnes, and B. F. Smith, PETSc Users Manual, Tech. Report ANL-95/11, Revision 2.1.5, Argonne National Laboratory, 2004.

[4] M. Benzi, Preconditioning techniques for large linear systems: A survey, J. Comput. Phys., 182 (2002), pp. 418-477.

[5] M. Benzi And G. H. Golub, A preconditioner for generalized saddle point problems, SIAM J. Matrix Anal. Appl., 26 (2004), pp. 20-41.

[6] M. Benzi, G. H. Golub, And J. Liesen, Numerical solution of saddle point problems, Acta Numer., 14 (2005), pp. 1-137.

[7] M. BEnZI AND M. TÜma, A sparse approximate inverse preconditioner for nonsymmetric linear systems, SIAM J. Sci. Comput., 19 (1998), pp. 968-994.

[8] M. Benzi And M. TŮma, A comparative study of sparse approximate inverse preconditioners, Appl. Numer. Math., 30 (1999), pp. 305-340.

[9] A. BRANDT, Multilevel computations of integral transforms and particle interactions with oscillatory kernels, Comput. Phys. Comm., 65 (1991), pp. 24-38.

[10] B. Carpentieri, I. S. Duff, L. Giraud, and G. Sylvand, Combining fast multipole techniques and an approximate inverse preconditioner for large electromagnetism calculations, SIAM J. Sci. Comput., 27 (2005), pp. 774-792.

[11] Y. Chang And R. F. HARRington, A surface formulation for characteristic modes of material bodies, IEEE Trans. Antennas and Propagation, 25 (1977), pp. 789-795.

[12] W. C. Chew, J.-M. Jin, E. Michielssen, And J. Song, eds., Fast and Efficient Algorithms in Computational Electromagnetics, Artech House, Norwood, MA, 2001.

[13] E. Chow AND Y. SAAD, Approximate inverse techniques for block-partitioned matrices, SIAM J. Sci. Comput., 18 (1997), pp. 1657-1675.

[14] E. Chow AND Y. SAAD, Experimental study of ILU preconditioners for indefinite matrices, J. Comput. Appl. Math., 86 (1997), pp. 387-414.

[15] E. Chow And Y. SAAD, Approximate inverse preconditioners via sparse-sparse iterations, SIAM J. Sci. Comput., 19 (1998), pp. 995-1023.

[16] E. De Sturler And J. Liesen, Block-diagonal and constraint preconditioners for nonsymmetric indefinite linear systems. Part I: Theory, SIAM J. Sci. Comput., 26 (2005), pp. 1598-1619.

[17] J. J. Dongarra, I. S. Duff, D. C. Sorensen, And H. A. van der Vorst, Numerical Linear Algebra for High-Performance Computers, SIAM, Philadelphia, 1998.

[18] D. A. Dunavant, High degree efficient symmetrical Gaussian quadrature rules for the triangle, Internat. J. Numer. Methods Engrg., 21 (1985), pp. 1129-1148.

[19] H. C. Elman, D. J. Silvester, And A. J. Wathen, Finite Elements and Fast Iterative Solvers: with Applications in Incompressible Fluid Dynamics, Oxford University Press, New York, 2005.

[20] Ö. ERGüL AND L. GüREL, Enhancing the accuracy of the interpolations and anterpolations in 
MLFMA, IEEE Trans. Antennas and Propagation Lett., 5 (2006), pp. 467-470.

[21] Ö. ERGüL AND L. Gürel, Optimal interpolation of translation operator in multilevel fast multipole algorithm, IEEE Trans. Antennas and Propagation, 54 (2006), pp. 3822-3826.

[22] Ö. ERGüL AND L. GÜREL, Comparison of integral-equation formulations for the fast and accurate solution of scattering problems involving dielectric objects with multilevel fast multipole algorithm, IEEE Trans. Antennas and Propagation, 57 (2009), pp. 176-187.

[23] J. M. Ford, K. Chen, AND D. Evans, On a recursive Schur preconditioner for iterative solution of a class of dense matrix problems, Int. J. Comput. Math., 80 (2003), pp. 105-122.

[24] G. H. Golub and C. F. Van Loan, Matrix Computations, 3rd ed., Johns Hopkins University Press, Baltimore, MD, 1996.

[25] R. D. Graglia, On the numerical integration of the linear shape functions times the 3-D Green's function or its gradient on a plane triangle, IEEE Trans. Antennas and Propagation, 41 (1993), pp. 1448-1455.

[26] M. J. Grote And T. Huckle, Parallel preconditioning with sparse approximate inverses, SIAM J. Sci. Comput., 18 (1997), pp. 838-853.

[27] L. GÜREL AND W. C. CHEw, Recursive algorithms for calculating the scattering from $N$ strips or patches, IEEE Trans. Antennas and Propagation, 38 (1990), pp. 507-515.

[28] L. Gürel AND W. C. Chew, A recursive T-matrix algorithm for strips and patches, Radio Sci., 27 (1992), pp. 387-401.

[29] L. GÜREL AND Ö. ERGÜL, Comparisons of FMM implementations employing different formulations and iterative solvers, in Proceedings of the IEEE Antennas and Propagation Society International Symposium, Vol. 1, 2003, pp. 19-22.

[30] L. GÜrel AND Ö. ErgüL, Singularity of the magnetic-field integral equation and its extraction, IEEE Antennas Wireless Propagat. Lett., 4 (2005), pp. 229-232.

[31] L. GÜREL AND T. MALAS, Iterative near-field preconditioner for the multilevel fast multipole algorithm, SIAM J. Sci. Comput., 32 (2010), pp. 1929-1949.

[32] W. W. HAGER, Updating the inverse of a matrix, SIAM Rev., 31 (1989), pp. 221-239.

[33] S. S. HaYkin, Kalman Filtering and Neural Networks, John Wiley \& Sons, New York, 2001.

[34] G. C. Hsiao and R. E. Kleinman, Mathematical foundations for error estimation in numerical solutions of integral equations in electromagnetics, IEEE Trans. Antennas and Propagation, 45 (1997), pp. 316-328.

[35] I. C. F. IPSEn, A note on preconditioning nonsymmetric matrices, SIAM J. Sci. Comput., 23 (2001), pp. 1050-1051.

[36] J. D. Joannopoulos, R. D. Meade, and J. N. Winn, Photonic Crystals: Molding the Flow of Light, Princeton University Press, Princeton, NJ, 2008.

[37] S. G. Johnson, S. Fan, P. R. Villeneuve, and J. D. Joannopoulos, Guided modes in photonic crystal slabs, Phys. Rev. B, 60 (1999), pp. 5751-5758.

[38] L. Y. Kolotilina And A. Y. Yeremin, Factorized sparse approximate inverse preconditioning I. Theory, SIAM J. Matrix Anal. Appl., 14 (1993), pp. 45-58.

[39] J. Lee, J. Zhang, And C.-C. Lu, Sparse inverse preconditioning of multilevel fast multipole algorithm for hybrid integral equations in electromagnetics, IEEE Trans. Antennas and Propagation, 52 (2004), pp. 158-175.

[40] L. LitTle, Y. SAAD, AND L. SMOch, Block LU preconditioners for symmetric and nonsymmetric saddle point problems, SIAM J. Sci. Comput., 25 (2003), pp. 729-748.

[41] T. W. Lloyd, J. M. Song, AND M. YANG, Numerical study of surface integral formulations for low-contrast objects, IEEE Antennas Wireless Propagat. Lett., 4 (2005), pp. 482-485.

[42] T. MALAS AND L. GÜREL, Incomplete LU preconditioning with the multilevel fast multipole algorithm for electromagnetic scattering, SIAM J. Sci. Comput., 29 (2007), pp. 1476-1494.

[43] T. Malas And L. Gürel, Accelerating the multilevel fast multipole algorithm with the sparseapproximate-inverse (SAI) preconditioning, SIAM J. Sci. Comput., 31 (2009), pp. 19681984.

[44] T. Malas And L. Gürel, Iterative Schur Complement Preconditioners for the Simulation of Dielectric Problems in Computational Electromagnetics, Tech. Report, Bilkent University, 2009.

[45] C. MüLLER, Foundations of the Mathematical Theory of Electromagnetic Waves, SpringerVerlag, New York, 1969.

[46] M. A. Olshanski And Y. V. Vassilevski, Pressure Schur complement preconditioners for the discrete Oseen problem, SIAM J. Sci. Comput., 29 (2007), pp. 2686-2704.

[47] A. P. Pavacic, D. L. Del Ro, J. R. Mosig, and G. V. Eleftheriades, Three-dimensional ray-tracing to model internal reflections in off-axis lens antennas, IEEE Trans. Antennas and Propagation, 54 (2006), pp. 604-612.

[48] A. J. Poggio ANd E. K. Miller, Integral equation solutions of three-dimensional scattering 
problems, in Computer Techniques for Electromagnetics, R. Mittra, ed., Pergamon Press, Oxford, 1973, Chap. 4, pp. 159-264.

[49] S. M. Rao, D. R. Wilton, and A. W. Glisson, Electromagnetic scattering by surfaces of arbitrary shape, IEEE Trans. Antennas and Propagation, 30 (1982), pp. 409-418.

[50] S. M. RAO AND D. R. Wilton, E-field, H-field, and combined-field solution for arbitrarily shaped three-dimensional dielectric bodies, Electromag., 10 (1990), pp. 407-421.

[51] Y. SAAD, Iterative Methods for Sparse Linear Systems, 2nd ed., SIAM, Philadelphia, 2003.

[52] G. Schmidlin, U. Fischer, Z. Andjeliĉ, And C. Schwab, Preconditioning of the second-kind boundary integral equations for $3 D$ eddy current problems, Internat. J. Numer. Methods Engrg., 51 (2001), pp. 1009-1031.

[53] X. Q. Sheng, J.-M. Jin, J. Song, W. C. Chew, and C.-C. Lu, Solution of combined-field integral equation using multilevel fast multipole algorithm for scattering by homogeneous bodies, IEEE Trans. Antennas and Propagation, 46 (1998), pp. 1718-1726.

[54] C. Siefert And E. DE Sturler, Preconditioners for generalized saddle-point problems, SIAM J. Numer. Anal., 44 (2006), pp. 1275-1296.

[55] V. Simoncini AND D. B. Szyld, The effect of non-optimal bases on the convergence of Krylov subspace methods, Numer. Math., 100 (2005), pp. 711-733.

[56] L. N. Trefethen and D. Bau, III, Numerical Linear Algebra, SIAM, Philadelphia, 1997.

[57] T.-K. Wu AND L. L. Tsai, Scattering from arbitrarily-shaped lossy dielectric bodies of revolution, Radio Sci., 12 (1977), pp. 709-718.

[58] P. Ylä-Oijala and M. TASKInen, Calculation of CFIE impedance matrix elements with RWG and $\hat{\boldsymbol{n}} \times R W G$ functions, IEEE Trans. Antennas and Propagation, 51 (2003), pp. 1837-1846.

[59] P. Ylä-Oijala and M. Taskinen, Application of combined field integral equation for electromagnetic scattering by composite metallic and dielectric objects, IEEE Trans. Antennas and Propagation, 53 (2005), pp. 1168-1173.

[60] P. Ylä-OiJala and M. TASKinen, Well-conditioned Muller formulation for electromagnetic scattering by dielectric objects, IEEE Trans. Antennas and Propagation, 53 (2005), pp. 3316-3323.

[61] P. Ylä-Oijala, M. Taskinen, and S. JäRvenpä̈̈, Surface integral equation formulations for solving electromagnetic scattering problems with iterative methods, Radio Sci., 40 (2005), article RS6002.

[62] P. Ylä-Oijala, M. Taskinen, and S. Järvenpä̈̈, Analysis of surface integral equations in electromagnetic scattering and radiation problems, Eng. Anal. Boundary Elem., 32 (2008), pp. 196-209.

[63] J. ZHANG, On preconditioning Schur complement and Schur complement preconditioning, Electron. Trans. Numer. Anal., 10 (2000), pp. 115-130.

Copyright $@$ by SIAM. Unauthorized reproduction of this article is prohibited. 